\title{
Determinantes de las exportaciones manufactureras en Argentina y México: un estudio comparativo
}

\section{Determinants of manufacture exports in Argentina and Mexico: a comparative study}

\author{
Víctor Manuel Cuevas-Ahumada*
}

\begin{abstract}
In this work we evaluate some determinants of manufacturing exports in Argentina and Mexico; to do so, two econometric methods are used: regression analysis, on the one side, and multivariate time series, on the other. A relevant finding is that in both nations, independently from the utilized econometric technique, labor productivity and external demand significantly influence on the referred exportations. The effects from other variables depend on the analyzed country andlor used econometric methodology; likewise, evidence suggests that a program of stimuli to labor productivity not only would increase exports to a larger extent than an actual exchange rate depreciation, but also would counteract the negative effects of an international recession.
\end{abstract}

Keywords: manufacturing exportations, labor productivity, econometric analysis, Argentina, Mexico.

\section{Resumen}

En este trabajo se evalúan algunos determinantes de las exportaciones manufactureras en Argentina y México. Para ello se recurre a dos métodos econométricos: análisis de regresión, por un lado, y análisis de series de tiempo multivariadas, por el otro. Un hallazgo relevante es que en ambas naciones y con independencia de la técnica econométrica utilizada, tanto la productividad laboral como la demanda externa influyen significativamente en las exportaciones referidas. Los efectos de otras variables dependen del país analizado y/o de la metodología econométrica empleada. Asimismo, la evidencia sugiere que un programa de estímulos a la productividad laboral no sólo aumentaría las exportaciones en mayor medida que una depreciación cambiaria real, sino que contrarrestaría los efectos negativos de una recesión internacional.

Palabras clave: exportaciones manufactureras, productividad laboral, análisis econométrico, Argentina, México.

* Universidad Autónoma Metropolitana, Azcapotzalco, México. Correo-e: victorcuevasahumada@yahoo.com.mx. 


\section{Introducción}

El propósito de esta investigación consiste en evaluar algunos de los principales determinantes de las exportaciones manufactureras, tomando como referencia las economías de Argentina y México. Estas naciones resultan interesantes para un análisis comparativo en virtud de la tendencia de crecimiento acelerado que han reportado, tanto en el rubro de productividad laboral como en el de exportaciones manufactureras, por lo menos, hasta el cierre de 2008. Como podrá recordarse, ambos países emprendieron reformas de orientación exportadora -con diferentes ritmos y alcances- en el marco del Consenso de Washington a partir de mediados de la década de los ochenta. Sin embargo, mientras que Argentina se integró regionalmente con países en desarrollo del Cono Sur, México optó por integrarse con dos economías desarrolladas y altamente competitivas en aras de explotar complementariedades en materia no sólo de estructuras de producción, sino también de factores productivos.

Con el objeto de obtener evidencia empírica más sólida, se hace uso de dos métodos econométricos: la regresión múltiple y las series de tiempo multivariadas. ${ }^{1}$ Estos dos métodos se complementan en razón de que el primero conduce a un análisis estático mediante la estimación de elasticidades, mientras que el segundo desemboca en un análisis dinámico por la vía de la estimación de funciones de impulso-respuesta. En cada caso, se asegura la estacionariedad de los datos y el adecuado comportamiento de los residuales mediante diversas pruebas de diagnóstico. Asimismo, para realizar el análisis dinámico se recurre a un modelo var generalizado (o modelo GVAR) para cada nación, cuya principal ventaja estriba en producir evidencia empírica que no depende de la ordenación de las ecuaciones del sistema. ${ }^{2}$

El hallazgo de mayor relevancia es que tanto la productividad laboral como la demanda externa influyen perceptiblemente en las exportaciones manufactureras. Esto se puede observar en ambos países y con independencia de la técnica econométrica utilizada. Los efectos de algunas otras variables sobre las exportaciones dependen del país analizado y/o de la metodología econométrica empleada. En el caso de México, el cuerpo de la evidencia empírica sugiere que el volumen de las exportaciones manufactureras puede resultar afectado por una depreciación del tipo de cambio real. Esto es consistente con la noción de que una depreciación

${ }^{1}$ El empleo de más de un método econométrico incrementa la confiabilidad de la evidencia empírica obtenida, puesto que se eliminan los sesgos asociados al uso de una técnica en particular (Walsh, 2003).

${ }^{2}$ En los modelos VAR recursivos tradicionales, por el contrario, tanto las funciones de impulsorespuesta como las descomposiciones de varianza son sensibles al orden en que se coloquen las variables involucradas. 
real, por una parte, fortalece la competitividad internacional por la vía del abaratamiento de las exportaciones en términos de dólares y, por otra, la debilita por la vía del encarecimiento de los insumos importados en moneda nacional. La interacción de estos dos efectos pareciera ser negativa para México, por lo menos, en el corto plazo. En el caso de Argentina, el tipo de cambio real no ejerce una influencia estadísticamente significativa en las exportaciones, lo cual es sintomático de que los dos efectos referidos se cancelan mutuamente. Por último, algunos de los hallazgos realizados son consistentes con la hipótesis de que las decisiones de endeudamiento, inversión, producción y contratación de personal de las empresas manufactureras están sobre todo orientadas a la expansión y diversificación de exportaciones.

En este contexto, tanto para Argentina como para México, hay dos importantes implicaciones de política económica y sectorial: la primera es que los efectos negativos de una recesión internacional sobre las exportaciones de manufacturas podrían compensarse (en buena medida) mediante aumentos en la productividad de los trabajadores; y la segunda es que las exportaciones manufactureras podrían responder mejor frente a un paquete integral y coherente de estímulo a la productividad del factor trabajo, que ante una depreciación del tipo de cambio real.

Este trabajo se divide en cinco secciones. En la primera se hace un breve análisis de la literatura reciente. En la segunda se define el modelo de referencia y se describen las variables utilizadas. En la tercera se determina el orden de integración para cada serie de tiempo, con el fin de lograr su estacionariedad mediante diferenciación cuando esto proceda. En la cuarta se estiman sendos modelos de regresión múltiple y se realizan diferentes pruebas de diagnóstico. En la quinta sección se especifican y estiman los modelos GVAR, se evalúan sus propiedades estadísticas y se realiza el análisis de sensibilidad para cada nación. Finalmente, se presentan las conclusiones del trabajo.

\section{Análisis de la literatura}

Con frecuencia, las funciones de exportación se especifican de acuerdo con modelos teóricos de demanda. En estos modelos el volumen exportado depende básicamente del tipo de cambio real y del nivel de ingreso foráneo, medido con algún indicador de actividad económica externa o mediante las importaciones realizadas por las naciones con las que se comercia. Sin embargo, ignorar factores de oferta en las ecuaciones de exportación produce sesgos en las estimaciones de la elasticidad precio e ingreso de las exportaciones (Riedel, 1988). En este contexto, la literatura reciente incluye tanto trabajos que apelan al modelo tradicional de 
demanda, como estudios que incorporan otras variables explicativas; en particular variables ligadas a la oferta de exportaciones y a la demanda doméstica de bienes exportables.

Con base en una muestra de 12 países en desarrollo (entre ellos, Argentina y México), se ha demostrado que las exportaciones son más sensibles frente a incrementos en la demanda externa que ante a variaciones en los precios relativos (Reinhart, 1995). ${ }^{3}$ En esta misma línea, al ampliar la muestra a 53 naciones, tanto desarrolladas como en vías de desarrollo, se ha concluido que en el largo plazo las elasticidades ingreso y precio son en promedio iguales a $1.5 \mathrm{y}-1$, respectivamente (Senhadji y Monetenegro, 1998).

En el caso concreto de Argentina, se ha logrado establecer: 1) que las exportaciones manufactureras responden en gran medida al nivel de actividad económica en el Mercosur y al tipo de cambio real del peso argentino frente al real brasileño, y 2) que una mayor inversión agregada neta estimularía las exportaciones, mientras que una expansión del consumo doméstico las inhibiría (Catao y Falsetti, 2002). ${ }^{4}$ También se han analizado -entre otras variables- el efecto del tipo de cambio real, la volatilidad cambiaria, la demanda externa y la capacidad instalada utilizada en las exportaciones manufactureras de origen industrial de la nación sudamericana. El hallazgo central es que la demanda externa influye más en las exportaciones referidas que el tipo de cambio real (Berrettoni y Castresana, 2007).

En el contexto de modelos de restricción externa al crecimiento, se han estimado funciones de exportación para México (Moreno-Brid, 1999; Loría, 2001). Por una parte, se ha colocado la tasa de crecimiento de la demanda de exportaciones como una función creciente del ingreso foráneo y decreciente de la tasa de variación en los términos de intercambio (Moreno-Brid, 1999). Por otra, se ha demostrado que las exportaciones tienen una relación directa de largo plazo con las siguientes variables: tipo de cambio real, nivel de actividad económica en Estados Unidos, y nivel de actividad económica en México (Loría, 2001)..$^{5}$

Continuando con el caso mexicano, hay evidencia de que las exportaciones manufactureras se ven afectadas no sólo por el tipo de cambio real y el nivel de actividad económica en Estados Unidos sino, también, por incrementos en la productividad del factor trabajo (Cuevas, 2008). En

${ }^{3}$ De allí que, de acuerdo con dicho estudio, se requiere de un ajuste considerable en el tipo de cambio real para impulsar de manera eficaz las exportaciones de un país.

${ }^{4}$ Esto en virtud de que la creciente demanda interna absorbería una porción de los bienes exportables.

${ }^{5}$ De acuerdo con este autor, los efectos positivos de la producción doméstica sobre las exportaciones totales pueden atribuirse a las economías de escala, externalidades y complementariedades que se generan conforme aumenta el tamańo de la economía. 
esta misma línea, una conclusión interesante es que la capacitación fortalece la competitividad de las manufacturas mexicanas, tanto en el ámbito de la firma como del sector (Padilla y Juárez, 2006). Por otra parte, se ha logrado identificar una relación estable de largo plazo entre las exportaciones totales de México, el índice de producción industrial estadounidense y el tipo de cambio real (Garcés, 2008).

Finalmente, algunos trabajos empíricos vinculan las exportaciones de las naciones en desarrollo con otras variables. Una de esas variables es la inversión extranjera directa (IED), la cual tiene efectos positivos en las exportaciones de los países de América Latina (Goldberg y Klein, 1997). ${ }^{6}$ Otro ejemplo destacado son costos laborales unitarios, los cuales guardan una relación inversa con las exportaciones de manufacturas en Senegal (Mbaye y Golub, 2002). Este hallazgo subrayaría la importancia de que, en aras de exportar más, la productividad laboral crezca por encima de los salarios (lo que, por definición, haría descender los costos del factor trabajo por unidad producida).

En síntesis, la evidencia econométrica acumulada sugiere que la elasticidad ingreso excede (en valor absoluto) a la elasticidad precio de la demanda de exportaciones. Por otro lado, las exportaciones de un país parecen depender no sólo del tipo de cambio real y la demanda foránea sino, también, de la demanda interna de mercancías exportables y de variables relacionadas con la oferta, como la productividad laboral, los salarios y la inversión extranjera directa.

\section{Definición del modelo}

El objetivo central de la investigación reside en comparar los efectos de diferentes variables sobre las exportaciones manufactureras de Argentina y México para, con fundamento en los hallazgos realizados, formular recomendaciones de política económica y sectorial. En este contexto, a reserva de realizar diversas pruebas para identificar variables redundantes y omitidas, se procede a estimar la siguiente función ampliada de exportaciones para cada país:

$$
X=f(v, W, Q, D E, I E D, R, C I U, P O)
$$

donde $X$ es volumen de exportaciones manufactureras totales, $v$ es la productividad de la mano de obra en la industria manufacturera, $W$ es el índice de salarios medios reales en la industria manufacturera, $Q$ es el

\footnotetext{
${ }^{6}$ Esta relación obedece a que los países receptores son utilizados como plataforma para exportar a naciones industrializadas; o bien, a que la IED favorece un comercio más amplio de insumos intermedios entre las empresas matrices y sus respectivas filiales.
} 
índice de tipo de cambio real efectivo, el cual es un tipo de cambio real multilateral, puesto que se basa en índices de precios y mide cambios en la competitividad internacional de una nación frente a sus principales socios comerciales, ${ }^{7} D E$ es la demanda externa de exportaciones manufactureras. En virtud de los resultados obtenidos mediante diferentes pruebas y estimaciones, y de que el comercio exterior argentino es mucho más diversificado que el mexicano, se optó por emplear diferentes variables proxy para la demanda externa de exportaciones de cada país. En el caso de México, se hace uso de las importaciones manufactureras totales de Estados Unidos. ${ }^{8}$ En el caso de Argentina, por otra parte, se recurre a las importaciones totales de manufacturas realizadas por un amplio conjunto de países; es decir, las 30 naciones que pertenecen a la Organización para la Cooperación y el Desarrollo Económico (OCDE) y los tres socios comerciales de Argentina en el ámbito del Mercado Común del Sur (Mercosur): Brasil, Paraguay y Uruguay. ${ }^{9}$ IED es la inversión extranjera directa. En aras de explicar mejor esta variable, en el caso de la economía argentina se opta por la IED en el sector privado no financiero. En el caso de la economía mexicana, por otra parte, se seleccionó la IED en el sector manufacturero. $R$ es la tasa de interés. Después de probar con diferentes alternativas de medición del costo del crédito, en el caso de Argentina se decidió utilizar el promedio aritmético de la tasa de interés por préstamos a empresas de primera línea, mientras que en el de México se recurrió a la tasa de interés promedio ponderada de los instrumentos de deuda a corto plazo. CIU es el porcentaje de capacidad instalada utilizada en la industria manufacturera. $P O$ es el índice de personal ocupado en dicha industria. La información del comportamiento de esta variable se encuentra disponible con la periodicidad requerida en el caso de México, mientras que en el de Argentina hubo necesidad de emplear el índice de obreros ocupados en calidad de variable proxy.

En el caso de México, el índice de tipo de cambio real efectivo mide los cambios en la competitividad internacional del país frente a 111 socios comerciales. En el caso de Argentina, el índice de tipo de cambio real multilateral mide los cambios en la competitividad internacional de esa nación frente a 18 socios comerciales.

${ }^{8}$ Ésta es una buena proxy para la demanda externa de manufacturas mexicanas, toda vez que Estados Unidos es el principal destino de exportación de México. En el año 2007, por ejemplo, $84.5 \%$ de las exportaciones mexicanas de mercancías se colocaron en ese país. Por otra parte, en el ańo referido, la participación de las exportaciones mexicanas de bienes en las importaciones de Estados Unidos fue de $10.8 \%$, lo cual coloca a México como el tercer proveedor más importante de mercancías en Estados Unidos después de Canadá y China (Informe Anual del Banco de México, 2007, p. 162).

${ }^{9}$ En 2007, las 33 naciones consideradas adquirieron $54.88 \%$ de las mercancías exportadas por Argentina, siendo Brasil el principal comprador puesto que, en ese país, se colocó $18.8 \%$ de las exportaciones totales (estimación propia con base en datos del Instituto Nacional de Estadística y Censos, INDEC, de la República Argentina). 
El abanico de variables explicativas responde a la disponibilidad de series estadísticas completas para las dos naciones, al propósito de capturar los principales efectos en las exportaciones manufactureras, a la evidencia empírica acumulada en el pasado reciente, y a la teoría económica. En primer lugar, hay que señalar que el tipo de cambio real $(Q)$ sirve para medir el efecto de los cambios en los precios relativos sobre las exportaciones. De acuerdo con la teoría prevaleciente, una depreciación (apreciación) real de la moneda incrementa (reduce) las exportaciones por la vía de su abaratamiento (encarecimiento) en términos de moneda foránea. La demanda externa $(D E)$, por su parte, permite capturar la relación directa entre los ingresos foráneos y las exportaciones del sector manufacturero.

La productividad laboral $(v)$ y los salarios $(W)$ determinan, conjuntamente, la evolución de los costos unitarios de la mano de obra en la industria manufacturera. Los incrementos en la productividad laboral, bajo la condición de ceteris paribus, fortalecen la competitividad internacional de las manufacturas por la vía de la reducción en los costos laborales unitarios. Contrariamente, los incrementos salariales tienden a elevar los costos del factor trabajo por unidad producida y erosionan, por ende, la competitividad internacional. De esta manera, los cambios en los salarios y en la productividad de los trabajadores afectan la competitividad internacional y, en consecuencia, los volúmenes exportados. ${ }^{10}$

La IED estimula las exportaciones manufactureras cuando las empresas extranjeras se establecen en México para aprovechar la abundancia de ciertos recursos (mano de obra o materias primas), reducir costos de producción y exportar productos a diferentes mercados. En este caso, México estaría atrayendo IED en función de los recursos y ventajas comparativas que ofrece para producir determinadas mercancías, por lo que serviría de plataforma para exportarlas a diferentes partes del mundo. En contraste, cuando las empresas extranjeras canalizan IED al país con el objeto de abastecer su mercado, la producción generada se orientará fundamentalmente al consumo interno y el potencial exportador será escaso. ${ }^{11}$

En esta misma línea, muchas empresas recurren al crédito para modernizarse y alcanzar mayores niveles de competitividad. Por tal motivo, una baja en la tasa de interés $(R)$ se traduce en un menor costo de adquisición de tecnología, maquinaria y equipo, mejorando - por esta vía- la competitividad internacional de las empresas. Asimismo, una fuente importante de innovaciones dentro de la empresa es la investigación y el desarrollo (I\&D). De esta manera, la I\&D pueden fortalecer la competitividad internacional y expandir las exportaciones de una empresa me-

\footnotetext{
${ }^{10}$ En relación con este punto, se sugiere leer el capítulo 3 de Pugel (2004).

${ }^{11}$ Para mayores detalles, véase Goldberg y Klein (1997).
} 
diante la reducción en los costos unitarios de producción o la mejora en la calidad del producto terminado. La tasa de interés constituye una variable relevante en este contexto, dado que las actividades I\&D normalmente entrañan costos e inversiones sustanciales.

Finalmente, habría que señalar que el porcentaje de capacidad instalada utilizada (CIU) y el índice de personal ocupado (PO) aparecen inicialmente en la ecuación (1) como variables de control. ${ }^{12}$ La inclusión de la CIU es conveniente para evitar que otras variables capturen los efectos que se producen, sobre las exportaciones del sector, conforme el PIв manufacturero efectivo (u observado) se aproxima o se aleja del pIB manufacturero potencial. De esta manera, se busca diferenciar los aumentos en las exportaciones derivados de una contracción de la demanda doméstica, la cual previsiblemente repercutiría en una menor CIU, de los aumentos en las exportaciones imputables a una mejora en la productividad laboral o a una expansión de la demanda externa. Asimismo, la incorporación del índice de po busca impedir que los parámetros de regresión asociados con la productividad laboral y con los salarios reflejen los efectos generados por el eventual despido de trabajadores, como consecuencia de la apertura comercial o de la adquisición de maquinaria, equipo y nuevas tecnologías. ${ }^{13}$

La ecuación (1) se estima en forma de regresión múltiple para, posteriormente, proceder a corregir eventuales problemas de sobreespecificación o subespecificación. Asimismo, se estima un modelo var generalizado para determinar si el análisis dinámico (es decir, las funciones de impulso-respuesta) es consistente con el análisis estático (es decir, con las elasticidades). Mediante el empleo de dos técnicas econométricas se tendrán diferentes perspectivas sobre la respuesta de las exportaciones manufactureras frente a incrementos en algunos de sus determinantes básicos: la productividad laboral, el tipo de cambio real y la demanda externa de exportaciones, entre otros.

Es importante precisar que, en el caso concreto de Argentina, no existen observaciones mensuales para algunas de las variables seleccionadas. De este modo, se procedió a recabar información estadística trimestral para cada variable de enero de 1998 a diciembre de 2008. ${ }^{14}$ Vale

${ }^{12}$ La incorporación de variables de control relevantes disminuye el riesgo de que los coeficientes de regresión estimados se encuentren sesgados debido a problemas de subespecificación del modelo.

${ }^{13}$ Véanse, por ejemplo, los trabajos de Jiménez et al. (1998), Catao y Falcetti (2002) y Berretoni y Castresana (2007).

${ }^{14}$ Los datos sobre la industrias manufactureras mexicana y argentina (es decir, exportaciones, productividad laboral, salarios, capacidad instalada utilizada y personal ocupado) provienen del INEGI y del INDEC, respectivamente. Esto mismo aplica al caso de las tasas de interés. Por otra parte, las series correspondientes al tipo de cambio real de cada nación se obtuvieron del Banco de México y del Banco Central de la República Argentina. La información sobre la IED proviene de la Secretaría de Economía, en lo referente a México, y del INDEC, en el caso de Argentina. Finalmente, con res- 
precisar que, con excepción de las tasas de interés de ambos países y de la IED correspondiente a Argentina, ${ }^{15}$ todas las series de tiempo utilizadas se encuentran expresadas en logaritmos naturales. Asimismo, todas las series (con excepción de la IED de Argentina) fueron ajustadas estacionalmente con el método de ajuste estacional Census X12-ARIMA. ${ }^{16}$

\section{Análisis de integración}

En virtud de la reciente proliferación de pruebas de raíz unitaria y de estacionariedad, y de que cada prueba tiene ventajas y desventajas particulares, aquí se utilizan tres pruebas diferentes para determinar el orden de integración de las series de tiempo: la Dickey-Fuller aumentada (o ADF, por sus siglas en inglés), la Phillips-Perron (PP) y la Kwiatkowski-PhillipsSchmidt-Shin (KPss). ${ }^{17}$ En los cuadros 1 y 2 aparecen los resultados de estas pruebas para las economías de Argentina y México, respectivamente.

Como se puede observar, las pruebas ADF y PP contrastan la hipótesis nula de raíz unitaria contra la alternativa de estacionariedad, mientras que la prueba KPSS contrasta la hipótesis nula de estacionariedad con la alternativa de raíz unitaria. ${ }^{18}$ Para especificar adecuadamente las ecuaciones de prueba (es decir, para decidir si procede incorporar una constante, una constante y una tendencia lineal, o ninguna de las cosas) se emplea la metodología propuesta por Hamilton (1994), que consiste en adoptar aquella especificación que mejor refleje el comportamiento de los datos, tanto bajo la hipótesis nula como bajo la hipótesis alternativa. ${ }^{19}$ Por otra parte, las pruebas formales adolecen necesariamente de bajo poder y con frecuencia arrojan resultados contradictorios, por lo que es conveniente estimar los modelos y evaluar (sobre todo en función de los coeficientes de determinación ajustados y del comportamiento de los residuales) si

pecto a México, la información concerniente a la demanda externa de exportaciones se obtuvo del Buró de Censos de Estados Unidos; mientras que en el caso de Argentina se recopiló de diferentes fuentes: la base de datos de la ocDE, el Ministerio de Desarrollo, Industria y Comercio Exterior de Brasil, y los Bancos Centrales de Paraguay y Uruguay.

${ }^{15}$ Las tasas de interés son variables que no tienden a crecer a lo largo del tiempo, por lo que no se acostumbra expresarlas en logaritmos naturales. Por otra parte, la IED correspondiente a la economía argentina asume valores negativos, por lo que no es posible expresarla en términos logarítmicos.

${ }^{16}$ Para la IED de Argentina se utilizó el método de ajuste estacional Tramo/Seats, dado que este método es apropiado para series de tiempo que asumen valores negativos o iguales a cero.

${ }^{17}$ Para una explicación detallada de estas pruebas, se recomienda consultar los capítulos 6 y 7 de Patterson (2000) y el trabajo de Kwiatkowski et al. (1992).

${ }^{18}$ De esta manera, para concluir que una variable es estacionaria sería necesario obtener un rechazo de la hipótesis nula en las pruebas ADF y PP y un no rechazo en la prueba KPss.

${ }^{19}$ En ciertos casos se realizaron pruebas de hipótesis tipo $t$ y $F$, empleando los valores críticos desarrollados por Dickey y Fuller (1981) y Dickey et al. (1986) para ese propósito, y se evaluó la sensibilidad del resultado obtenido frente a especificaciones alternativas. 
Cuadro 1

Pruebas de raíz unitaria y de estacionariedad para Argentina

\begin{tabular}{|c|c|c|c|c|c|}
\hline Variable & $\begin{array}{l}\text { Especifi- } \\
\text { cación }\end{array}$ & $\begin{array}{c}\text { Estadistica } \\
\text { ADF } \\
\text { Ho: raiz } \\
\text { unitaria }\end{array}$ & $\begin{array}{c}\text { Estadistica } \\
P P \\
\text { Ho: raiz } \\
\text { unitaria }\end{array}$ & $\begin{array}{c}\text { Estadistica } \\
\text { KPSS } \\
\text { Ho: } \\
\text { estaciona- } \\
\text { riedad }\end{array}$ & $\begin{array}{c}\text { Orden de } \\
\text { integración }\end{array}$ \\
\hline$X_{t}$ & С у TD & 2.39 & 2.85 & $0.23^{* *}$ & $\mathrm{I}(2)$ o $\mathrm{I}(1)$ \\
\hline$\Delta X_{t}$ & $\mathrm{C}$ & -0.53 & $-4.14^{* *}$ & $0.72^{*}$ & $\mathrm{I}(1)$ o $\mathrm{I}(0)$ \\
\hline$\Delta^{2} X_{t}^{t}$ & $\mathrm{C}$ & $-8.79^{* *}$ & $-7.68^{* *}$ & 0.41 & $\mathrm{I}(0)$ \\
\hline$\Delta^{2} X_{t}^{t}$ & Sin $\mathrm{C}$ ni $\mathrm{TD}$ & $-8.86^{* *}$ & $-7.71^{* *}$ & n.d. & $\mathrm{I}(0)$ \\
\hline$v_{t}$ & C у TD & -2.23 & -2.30 & 0.13 & $\mathrm{I}(1)$ o I $(0)$ \\
\hline$\Delta v_{t}$ & $\mathrm{C}$ & $-4.81^{* *}$ & $-4.82^{* *}$ & 0.17 & $\mathrm{I}(0)$ \\
\hline$W_{t}^{t}$ & C y TD & -1.05 & -0.72 & $0.58^{* *}$ & $\mathrm{I}(2)$ o $\mathrm{I}(1)$ \\
\hline$\Delta W_{t}$ & $\mathrm{C}$ & $-2.97^{* *}$ & $-2.97^{* *}$ & $0.53^{*}$ & $\mathrm{I}(1)$ o $\mathrm{I}(0)$ \\
\hline$\Delta^{2} \stackrel{t}{W}_{t}$ & $\mathrm{C}$ & $-7.91^{* *}$ & $-7.97^{* *}$ & 0.41 & $\mathrm{I}(0)$ \\
\hline$\Delta^{2} W_{t}^{t}$ & Sin $\mathrm{C}$ ni TD & $-7.99^{* *}$ & $-7.99^{* *}$ & n.d. & $\mathrm{I}(0)$ \\
\hline$Q_{t}$ & $\mathrm{C}$ & 1.55 & -1.12 & 0.38 & $\mathrm{I}(1)$ o I $(0)$ \\
\hline$\Delta Q$ & $\mathrm{C}$ & $-4.34^{* *}$ & $-4.43^{* *}$ & 0.08 & $\mathrm{I}(0)$ \\
\hline$\Delta Q_{t}$ & Sin $\mathrm{C}$ ni $\mathrm{TD}$ & $-4.34^{* *}$ & $-4.43^{* *}$ & n.d. & $\mathrm{I}(0)$ \\
\hline$D E_{t}^{t}$ & C у TD & -1.41 & -0.94 & $0.43^{*}$ & $\mathrm{I}(1)$ \\
\hline$\Delta D E_{t}$ & $\mathrm{C}$ & $-3.70^{* *}$ & $-3.70^{* *}$ & 0.46 & $\mathrm{I}(0)$ \\
\hline$I E D_{t}{ }^{t}$ & $\mathrm{C}$ & -1.60 & $-3.71^{* *}$ & 0.39 & $\mathrm{I}(1)$ o $\mathrm{I}(0)$ \\
\hline$\Delta I E D_{t}$ & $\mathrm{C}$ & $-14.51^{* *}$ & $-14.75^{* *}$ & 0.16 & $\mathrm{I}(0)$ \\
\hline$\triangle I E D_{t}$ & Sin $\mathrm{C}$ ni $\mathrm{TD}$ & $-14.67^{* *}$ & $-14.92^{* *}$ & n.d. & $\mathrm{I}(0)$ \\
\hline$R_{t}{ }^{t}$ & $\mathrm{C}$ & -2.18 & -2.29 & 0.11 & $\mathrm{I}(1)$ o $\mathrm{I}(0)$ \\
\hline$\Delta R_{t}$ & $\mathrm{C}$ & $-6.44^{* *}$ & $-6.44^{* *}$ & 0.06 & $\mathrm{I}(0)$ \\
\hline$\Delta R_{t}^{t}$ & Sin $\mathrm{C}$ ni TD & $-6.50^{* *}$ & $-6.50^{* *}$ & n.d. & $\mathrm{I}(0)$ \\
\hline$C I U_{t}$ & C у TD & $-4.01^{* *}$ & $-6.40^{* *}$ & $0.17^{*}$ & $\mathrm{I}(2)$ o $\mathrm{I}(1)$ \\
\hline$\Delta C I U_{t}$ & $\mathrm{C}$ & $-5.37^{* *}$ & $-5.34^{* *}$ & $0.60^{*}$ & $\mathrm{I}(1)$ o $\mathrm{I}(0)$ \\
\hline$\Delta^{2} C I U_{t}^{t}$ & $\mathrm{C}$ & $-11.86^{* *}$ & $-22.18^{* *}$ & 0.18 & $\mathrm{I}(0)$ \\
\hline$\Delta^{2} C I U_{t}^{t}$ & Sin $\mathrm{C}$ ni $\mathrm{TD}$ & $-12.01^{* *}$ & $-16.04^{* *}$ & n.d. & $\mathrm{I}(0)$ \\
\hline $\mathrm{PO}_{t}{ }^{t}$ & C & -2.23 & -1.62 & $0.64^{*}$ & $\mathrm{I}(2)$ \\
\hline$\Delta P O_{t}$ & $\mathrm{C}$ & -1.47 & -1.59 & $0.51^{*}$ & $\mathrm{I}(1)$ \\
\hline$\Delta^{2} P O_{t}^{t}$ & $\mathrm{C}$ & $5.57^{* *}$ & $5.52^{* *}$ & 0.09 & $\mathrm{I}(0)$ \\
\hline$\Delta^{2} P O_{t}$ & Sin $\mathrm{C}$ ni TD & $-5.63^{* *}$ & $-5.63^{* *}$ & n.d. & $\mathrm{I}(0)$ \\
\hline
\end{tabular}

Notas:

1. Los símbolos $\Delta \mathrm{y} \Delta^{2}$ son los operadores de primeras y segundas diferencias, respectivamente.

De este modo, $\Delta Q_{t}=Q_{t}-Q_{t-1}$.

2. C es constante y TD tendencia determinística.

3. Los asteriscos ${ }^{*} \mathrm{y}^{* *}$ indican el rechazo de la hipótesis nula a un nivel de significancia de 5 y $1 \%$, respectivamente.

4. n.d.: no disponible.

5. Tanto en las pruebas ADF como en las pp se utilizan los valores críticos de MacKinnon (1996). Dichos valores, por supuesto, varían en función de la especificación de la ecuación de prueba.

6. En las pruebas ADF se recurre al criterio de información de Schwarz para determinar el número de rezagos de las ecuaciones de prueba.

7. Los resultados de las pruebas KPss se basan en los valores críticos tabulados por Kwiatkowski, Phillips, Schmidt y Shin (1992).

Fuente: Elaboración propia. 
Cuadro 2

Pruebas de raíz unitaria y de estacionariedad para México

\begin{tabular}{|c|c|c|c|c|c|}
\hline Variable & $\begin{array}{l}\text { Especifi- } \\
\text { cación }\end{array}$ & $\begin{array}{c}\text { Estadistica } \\
\text { ADF } \\
\text { Ho: raiz } \\
\text { unitaria }\end{array}$ & $\begin{array}{c}\text { Estadistica } \\
P P \\
\text { Ho: raiz } \\
\text { unitaria }\end{array}$ & $\begin{array}{c}\text { Estadistica } \\
\text { KPSS } \\
\text { Ho: } \\
\text { estaciona- } \\
\text { riedad }\end{array}$ & $\begin{array}{c}\text { Orden de } \\
\text { integración }\end{array}$ \\
\hline$X_{t}$ & C y TD & -1.97 & -1.97 & 0.11 & $\mathrm{I}(1)$ o $\mathrm{I}(0)$ \\
\hline$\Delta X_{t}$ & $\mathrm{C}$ & $-4.66^{* *}$ & $-4.63^{* *}$ & 0.11 & $\mathrm{I}(0)$ \\
\hline$v_{t}$ & C y TD & -1.75 & -1.92 & 0.13 & $\mathrm{I}(1)$ o $\mathrm{I}(0)$ \\
\hline$\Delta v_{t}$ & C & $-6.53^{* *}$ & $-6.45^{* *}$ & 0.28 & $\mathrm{I}(0)$ \\
\hline$W_{t}^{t}$ & C y TD & 1.24 & 0.54 & $0.23^{* *}$ & $\mathrm{I}(1)$ \\
\hline$\Delta W_{t}$ & $\mathrm{C}$ & $-5.97^{* *}$ & $-6.30^{* *}$ & 0.44 & $\mathrm{I}(0)$ \\
\hline$Q_{t}$ & C & -1.90 & -2.84 & 0.34 & $\mathrm{I}(2)$ o $\mathrm{I}(1)$ \\
\hline$\Delta Q_{t}$ & $\mathrm{C}$ & $-4.89^{* *}$ & $-4.97^{* *}$ & $0.52^{*}$ & $\mathrm{I}(1)$ o $\mathrm{I}(0)$ \\
\hline$\Delta Q_{t}$ & Sin $\mathrm{C}$ ni TD & $-4.95^{* *}$ & $-5.02^{* *}$ & n.d. & $\mathrm{I}(0)$ \\
\hline$D E_{t}$ & с у TD & -3.31 & -2.13 & 0.11 & $\mathrm{I}(1)$ o $\mathrm{I}(0)$ \\
\hline$\Delta D E_{t}$ & $\mathrm{C}$ & $-4.35^{* *}$ & $-4.99^{* *}$ & 0.06 & $\mathrm{I}(0)$ \\
\hline $\mathrm{IED}_{\mathrm{t}}$ & C & $-5.48^{* *}$ & $-5.50^{* *}$ & 0.14 & $\mathrm{I}(0)$ \\
\hline$R_{t}$ & C & -2.13 & -1.69 & $0.69^{*}$ & $\mathrm{I}(1)$ \\
\hline$\Delta R_{t}$ & $\mathrm{C}$ & $-3.64^{* *}$ & $-3.08^{*}$ & 0.13 & $\mathrm{I}(0)$ \\
\hline$\Delta R_{t}^{t}$ & Sin $\mathrm{C}$ ni $\mathrm{TD}$ & $-3.65^{* *}$ & $-3.26^{* *}$ & n.d. & $\mathrm{I}(0)$ \\
\hline$C I U_{t}$ & $\mathrm{C}$ & -1.31 & -1.40 & $0.57^{*}$ & $\mathrm{I}(1)$ \\
\hline$\Delta C I U_{t}$ & $\mathrm{C}$ & $-6.80^{* *}$ & $-6.81^{* *}$ & 0.09 & $\mathrm{I}(0)$ \\
\hline$\Delta C I U_{t}^{t}$ & Sin $\mathrm{C}$ ni $\mathrm{TD}$ & $-6.82^{* *}$ & $-6.83^{* *}$ & n.d. & $\mathrm{I}(0)$ \\
\hline $\mathrm{PO}_{t}$ & C & -1.07 & -0.52 & $0.61^{*}$ & $\mathrm{I}(1)$ \\
\hline$\Delta P O_{t}$ & $\mathrm{C}$ & -2.06 & -2.04 & 0.33 & $\mathrm{I}(1)$ o $\mathrm{I}(0)$ \\
\hline$\Delta P O_{t}$ & Sin $\mathrm{C}$ ni TD & $-1.97^{*}$ & $-1.97^{*}$ & n.d. & $\mathrm{I}(0)$ \\
\hline
\end{tabular}

Notas:

1. Los símbolos $\Delta \mathrm{y} \Delta^{2}$ son los operadores de primeras y segundas diferencias, respectivamente. De este modo, $\Delta Q_{t}=Q_{t}-Q_{t-1}$.

2. C es constante y TD tendencia determinística.

3. Los asteriscos ${ }^{*} \mathrm{y}^{* *}$ indican el rechazo de la hipótesis nula a un nivel de significancia de 5 y $1 \%$, respectivamente.

4. n.d.: no disponible.

5. Tanto en las pruebas ADF como en las pp se utilizan los valores críticos de MacKinnon (1996). Dichos valores, por supuesto, varían en función de la especificación de la ecuación de prueba.

6. En las pruebas ADF se recurre al criterio de información de Schwarz para determinar el número de rezagos de las ecuaciones de prueba.

7. Los resultados de las pruebas KPsS se basan en los valores críticos tabulados por Kwiatkowski, Phillips, Schmidt y Shin (1992).

Fuente: Elaboración propia.

una variable determinada debe ingresar en niveles, en primeras o en segundas diferencias. ${ }^{20}$

En lo concerniente a Argentina, hay cuatro variables que podrían tratarse como integradas de orden 2 (o I (2)): las exportaciones manufac-

${ }^{20}$ De acuerdo con Gujarati (2004: 779), la mayoría de las variables económicas son I(1); es decir, deben diferenciarse una vez para volverse estacionarias. 
tureras $(X)$, los salarios (W), la capacidad instalada utilizada (CIU) y el personal ocupado (PO). Las exportaciones manufactureras, inclusive, registran un crecimiento explosivo a partir del año 2002, lo cual explicaría el hecho de que las pruebas ADF y PP arrojen una estadística positiva (igual a 2.39 y 2.85 , respectivamente) cuando se trabaja en niveles. ${ }^{21} \mathrm{Al}$ sacar primeras diferencias, los resultados de las pruebas se tornan contradictorios y solamente coinciden después de obtener segundas diferencias. Algo similar ocurre con los salarios y la capacidad instalada utilizada, mientras que con el personal ocupado las pruebas son consistentes al indicar que se trata de una variable I(2). En el Apéndice i se puede apreciar que las gráficas de estas cuatro variables registran patrones de crecimiento acelerado de 2002 en adelante. Aun cuando esto sugeriría la posible presencia de dos raíces unitarias, los resultados aquí propuestos deben tomarse con reserva en virtud de que se trabaja con una muestra pequeña.

El resto de las variables de la economía argentina, es decir, la productividad laboral $(v)$, el tipo de cambio real $(Q)$, la demanda externa (DE), la inversión extranjera directa (IED) y la tasa de interés $(R)$, serán tratadas como variables I(1). Esta decisión es procedente puesto que, a pesar de algunas inconsistencias en los resultados, en términos generales la evidencia apunta a que estas variables son I(1). Vale precisar, sin embargo, que en el caso específico de la IED el margen de duda es un poco mayor en razón de que las pruebas de raíz unitaria se contradicen entre sí: la prueba ADF sugiere la no estacionariedad de la serie, mientras que la prueba pP sugiere lo contrario. Esta divergencia podría deberse a que, cuando se trabaja con muestras finitas como en este caso, la prueba pp tiene un desempeño inferior a la ADF. ${ }^{22}$ Por lo que se refiere a la prueba KPSS, aunque no se alcanza a rechazar la hipótesis nula de estacionariedad a un nivel de significancia de 5\%, la estadística de prueba permite rechazar holgadamente esta hipótesis a un nivel de significancia de $10 \% .{ }^{23}$ De allí que, en los modelos estimados, la IED ingrese en primeras diferencias para tener una seguridad razonable de que sus características estocásticas no se modifican con el paso del tiempo.

En lo referente a México, la IED es claramente una variable estacionaria (o I $(0)$ ) en niveles, no sólo en virtud de los resultados de la pruebas formales sino del análisis de su función de autocorrelación estimada. El resto de las variables contempladas serán tratadas como I(1) en niveles y

${ }^{21}$ Esto constituye un resultado atípico, pero puede ocurrir cuando una serie de tiempo se incrementa a una tasa creciente. Haciendo uso del filtro de Hodrick-Prescott, es posible observar el cambio gradual en la tendencia de crecimiento de las exportaciones argentinas durante el periodo considerado.

${ }^{22}$ Para mayores detalles al respecto, véase Schwert (1989) y Perron y Ng (1996).

${ }^{23}$ Dado que el valor crítico asociado con un nivel de significancia de $10 \%$ es igual a 0.347 , el cual resulta inferior a la estadística de prueba (0.391). 
estacionarias en primeras diferencias. Como se puede ver, en el caso de México algunas series de tiempo exhiben un menor orden de integración, lo cual es consistente con el hecho de que esta nación gozó de una mayor estabilidad macroeconómica que Argentina en el periodo de referencia: 1998-2008. En segundo lugar, a pesar de que no todas las pruebas formales relativas a México apuntan a un mismo resultado, la revisión del conjunto de la evidencia empírica sugiere que, con excepción de la IED, todas las variables deben diferenciarse una sola vez para asegurar su estacionariedad. Esta apreciación se fortalece no sólo al analizar los correlogramas de cada serie de tiempo, sino también al estimar el modelo de regresión y corroborar que el comportamiento de los residuales mejora una vez que se trabaja con variables estacionarias.

\section{Modelos estacionarios de regresión múltiple y pruebas de diagnóstico}

El siguiente paso consiste en estimar sendas regresiones múltiples para Argentina y México mediante el método de mínimos cuadrados ordinarios (MCO), en realizar las pruebas de diagnóstico y las correcciones pertinentes, y en interpretar los resultados obtenidos. Es importante hacer notar que las series de tiempo han sido diferenciadas con el objeto de volverlas estacionarias. Como podrá recordarse, algunas variables de la economía argentina resultaron I(2), por lo que hubo necesidad de expresarlas en segundas diferencias para garantizar que sus propiedades estocásticas no fueran una función del tiempo. En lo concerniente a México se concluyó que, con excepción de la IED, todas las variables deben tratarse como I(1); de allí que la IED ingrese a la ecuación de regresión en niveles mientras que el resto de las variables lo haga en primeras diferencias. En este contexto, las ecuaciones de regresión estimadas para cada país se muestran en los cuadros 3 y 4 .

Como podrá recordarse, todas las variables (con excepción de la tasa de interés de ambos países y la IED correspondiente a Argentina) se encuentran expresadas en logaritmos naturales, por lo que los coeficientes de regresión estimados deben interpretarse como elasticidades. Vale señalar, por otra parte, que una eventual subespecificación del modelo ocasionaría un sesgo en los estimadores de MCO, así como inconsistencia en la medida en que el sesgo persistiera en muestras grandes. Contrariamente, una eventual sobreespecificación del modelo se traduciría en problemas de multicolinealidad y falta de eficiencia, lo que dificultaría la identificación de relaciones estadísticamente significativas. ${ }^{24}$ En este contexto, antes de

\footnotetext{
${ }^{24}$ Esto en virtud de que la multicolinealidad incrementa las varianzas estimadas de los parámetros estimados y, por tanto, disminuye las estadísticas $t$ en valor absoluto.
} 


\section{Cuadro 3 \\ Ecuación de las exportaciones manufactureras correspondiente a Argentina}

\begin{tabular}{|c|c|c|c|c|}
\hline \multicolumn{5}{|c|}{ Variable dependiente: $\Delta^{2} X_{t}$} \\
\hline Regresores & Coeficiente & Error estándar & Estadistica $t$ & $\begin{array}{c}\text { Valor de } \\
\text { probabilidad }\end{array}$ \\
\hline$\Delta v_{t}$ & 1.705927 & 0.393696 & 4.333106 & 0.0006 \\
\hline$\Delta^{2} W_{t}$ & 0.207809 & 0.231148 & 0.899033 & 0.3828 \\
\hline$\Delta Q_{t}$ & -0.323485 & 0.202320 & -1.598878 & 0.1307 \\
\hline$\Delta D E_{t}$ & 0.943737 & 0.354032 & 2.665681 & 0.0176 \\
\hline$\Delta \mathrm{IED}_{\mathrm{t}}$ & 0.000126 & $3.65 \mathrm{E}-05$ & 3.444622 & 0.0036 \\
\hline$\Delta R_{t}$ & 0.002428 & 0.000638 & 3.806541 & 0.0017 \\
\hline$\Delta^{2} C I U_{t}$ & -0.375070 & 0.208596 & -1.798066 & 0.0923 \\
\hline$\Delta^{2} P O_{t}$ & 0.760451 & 0.940180 & 0.808835 & 0.4313 \\
\hline Intercepto & -0.063261 & 0.019769 & -3.200000 & 0.0060 \\
\hline$R^{2}$ ajustada & 0.777926 & \multicolumn{2}{|c|}{ Error estándar de la regresión } & 0.029698 \\
\hline Estadística DW & 1.562537 & \multicolumn{2}{|c|}{ Valor de prob. de la prueba $F$} & 0.000048 \\
\hline
\end{tabular}

Fuente: Elaboración propia.

\section{Cuadro 4}

\section{Ecuación de las exportaciones manufactureras correspondiente a México}

\begin{tabular}{|c|c|c|c|c|}
\hline \multicolumn{5}{|c|}{ Variable dependiente: $\Delta^{2} X_{t}$} \\
\hline Regresores & Coeficiente & Error estándar & Estadistica $t$ & $\begin{array}{c}\text { Valor de } \\
\text { probabilidad }\end{array}$ \\
\hline$\Delta v_{t}$ & 0.734544 & 0.363798 & 2.019097 & 0.0508 \\
\hline$\Delta^{2} W_{t}$ & -0.460301 & 0.227639 & -2.022066 & 0.0504 \\
\hline$\Delta Q_{t}$ & -0.221444 & 0.096865 & -2.286113 & 0.0281 \\
\hline$\Delta D E_{t}$ & 0.338976 & 0.154236 & 2.197775 & 0.0343 \\
\hline$\Delta \mathrm{IED}_{\mathrm{t}}$ & -0.008073 & 0.009468 & -0.852709 & 0.3993 \\
\hline$\Delta R_{t}$ & -0.000333 & 0.001849 & -0.179935 & 0.8582 \\
\hline$\Delta^{2} C I U_{t}$ & 0.331298 & 0.610764 & 0.542432 & 0.5908 \\
\hline$\Delta^{2} P O_{t}$ & 0.966527 & 0.463234 & 2.086476 & 0.0439 \\
\hline Intercepto & 0.074472 & 0.080267 & 0.927802 & 0.3595 \\
\hline$R^{2}$ ajustada & 0.493972 & \multicolumn{2}{|c|}{ Error estándar de la regresión } & 0.020655 \\
\hline Estadística DW & 2.391442 & \multicolumn{2}{|c|}{ Valor de prob. de la prueba $F$} & 0.000029 \\
\hline
\end{tabular}

Fuente: Elaboración propia. 
proceder a afinar la especificación del modelo, conviene señalar que tanto en México como en Argentina los parámetros asociados a la productividad laboral $(v)$ y a la demanda externa (DE) son positivos y estadísticamente significativos (a un nivel de significancia de 5 por ciento). ${ }^{25}$

\subsection{Reespecificación de las ecuaciones de regresión}

Para identificar variables (o subconjuntos de variables) potencialmente redundantes u omitidas, ${ }^{26}$ se realizaron diversas pruebas de hipótesis basadas en el cociente de verosimilitud. En este caso, la decisión de excluir o de incorporar una o más variables depende de la diferencia entre la verosimilitud logarítmica del modelo restringido y la del no restringido. Bajo la hipótesis nula, esta diferencia sigue una distribución $\chi^{2}$ con un número de grados de libertad igual al número de restricciones impuestas. ${ }^{27}$ Después de realizar diferentes pruebas de hipótesis, tanto individuales como conjuntas, se llegó a las ecuaciones de regresión reformuladas que aparecen en los cuadros 5 y 6 .

En relación con Argentina (cuadro 5) sucede que el tipo de cambio real, los salarios y el personal ocupado a final de cuentas no resultaron estadísticamente diferentes de cero y tuvieron que excluirse de la ecuación. Esto sugiere que, por lo menos en el corto plazo, una depreciación real de la moneda no incide de manera ostensible en el volumen exportado; posiblemente, debido a que se requiere tiempo para establecer nuevos canales de distribución y para ampliar la capacidad instalada. Otra posibilidad es que el abaratamiento de los productos exportados asociado a la depreciación real se vea contrarrestado por el encarecimiento (en moneda nacional) de los insumos importados. De esta manera, la depreciación de la moneda estaría generando efectos contrapuestos en la competitividad internacional, por lo que el tipo de cambio real no afectaría en volumen exportado.

Asimismo, las variables que permanecen en la ecuación de regresión permiten hacer hallazgos interesantes para la economía de la nación sudamericana. En primer lugar, los parámetros asociados a la productividad

\footnotetext{
${ }^{25}$ Otras variables alcanzan significancia estadística en una u otra nación pero, por razones de espacio, esta evidencia empírica se interpretará cuando se aborden los modelos de regresión reespecificados.

${ }^{26}$ Se intentó incorporar algunas variables adicionales a las ecuaciones de regresión referidas, como la volatilidad del tipo de cambio real, la inversión fija bruta, o variables dicotómicas para capturar episodios de volatilidad macroeconómica, pero éstas en general no alcanzaron significancia estadística ni mejoraron el ajuste de curva. En algunos casos incluso hubo pérdida de eficiencia y desmejoró el comportamiento de los residuales. La única excepción, como podrá verse, fue la variable dicotómica que se incorpora en el caso de Argentina (véase el cuadro 5).

${ }^{27}$ En relación con esta temática, es conveniente consultar la sección 16.6 del libro de Greene (2008).
} 


\section{Cuadro 5}

\section{Ecuación reespecificada de las exportaciones manufactureras correspondiente a Argentina}

\begin{tabular}{|c|c|c|c|c|}
\hline \multicolumn{5}{|c|}{ Variable dependiente: $\Delta^{2} X_{t}$} \\
\hline Regresores & Coeficiente & Error estándar & Estadistica $t$ & $\begin{array}{c}\text { Valor de } \\
\text { probabilidad }\end{array}$ \\
\hline$\Delta v_{t}$ & 1.930822 & 0.335830 & 5.749396 & 0.0000 \\
\hline$\Delta D E_{t}$ & 1.017330 & 0.321347 & 3.165829 & 0.0056 \\
\hline$\Delta \mathrm{IED}_{\mathrm{t}}$ & 0.000111 & $3.22 \mathrm{E}-05$ & 3.441916 & 0.0031 \\
\hline$\Delta R_{t}$ & 0.001697 & 0.000542 & 3.127890 & 0.0061 \\
\hline$\Delta^{2} C I U_{t}$ & -0.634734 & 0.205923 & -3.082378 & 0.0068 \\
\hline$D_{t}$ & -0.043404 & 0.019318 & -2.246790 & 0.0382 \\
\hline Intercepto & -0.054134 & 0.013353 & -4.053960 & 0.0008 \\
\hline$R^{2}$ ajustada & 0.814827 & \multicolumn{2}{|c|}{ Error estándar de la regresión } & 0.027119 \\
\hline Estadística DW & 1.630399 & \multicolumn{2}{|c|}{ Valor de prob. de la prueba $F$} & 0.000002 \\
\hline
\end{tabular}

Nota: $D_{t}$ es una variable dicotómica para capturar los efectos de la crisis económica argentina de diciembre de 2001, así como el episodio de volatilidad cambiaria de finales de 2007 y principios de 2008. De allí que dicha variable asuma el valor 1 en los tres primeros trimestres de 2002 y en el primer trimestre de 2008, y cero en el resto del periodo.

Fuente: Elaboración propia.

\section{Cuadro 6}

\section{Ecuación reespecificada de las exportaciones manufactureras correspondiente a México}

\begin{tabular}{|c|c|c|c|c|}
\hline \multicolumn{5}{|c|}{ Variable dependiente: } \\
\hline Regresores & Coeficiente & Error estándar & Estadisticat & $\begin{array}{c}\text { Valor de } \\
\text { probabilidad }\end{array}$ \\
\hline$\Delta v_{t}$ & 0.763204 & 0.328238 & 2.325152 & 0.0254 \\
\hline$\Delta Q_{t}$ & -0.209810 & 0.076466 & -2.743843 & 0.0091 \\
\hline$\Delta D E_{\mathrm{t}}$ & 0.486767 & 0.125074 & 3.891840 & 0.0004 \\
\hline$\Delta P O_{t}$ & 0.875838 & 0.478531 & 1.830264 & 0.0749 \\
\hline Intercepto & -0.000190 & 0.005261 & -0.036093 & 0.9714 \\
\hline$R^{2}$ ajustada & 0.509208 & \multicolumn{2}{|c|}{ Error estándar de la regresión } & 0.021347 \\
\hline Estadística DW & 2.428427 & \multicolumn{2}{|c|}{ Valor de prob. de la prueba $F$} & 0.000002 \\
\hline
\end{tabular}

Fuente: Elaboración propia.

laboral y a la demanda externa son positivos y estadísticamente significativos a un nivel de significancia de $1 \%$. De acuerdo con estos resultados, una mayor tasa de crecimiento de la productividad, o un mayor dinamismo de los mercados foráneos, provocaría una expansión de las exportaciones manufactureras. Alternativamente, un programa de políticas sectoriales activas de aliento a la productividad de los trabajadores podría contra- 
rrestar, en buena medida, los efectos negativos de una recesión internacional sobre las exportaciones de manufacturas.

En segundo lugar, el coeficiente de la IED es positivo y estadísticamente significativo a un nivel de 1\%. Una explicación tentativa para este resultado es que Argentina al fin está logrando consolidar, de manera gradual, un proceso de industrialización basado en las manufacturas dinámicas; es decir, las encargadas de producir bienes con un cierto grado de contenido tecnológico para los mercados de exportación. Todavía a mediados de los noventa, la IED que llegó a Argentina buscaba fundamentalmente abastecer el mercado local o subregional, pero ahora parecería estar en curso un proceso de transformación estructural en el que la IED se canaliza, cada vez en mayor medida, a hacer más eficientes los sistemas de producción internacionalmente integrados de las grandes transnacionales con miras a exportar productos a diferentes partes del mundo. ${ }^{28}$

El parámetro de la tasa de interés también es estadísticamente significativo a un nivel de $1 \%$, pero presenta un signo positivo, lo cual contradice la teoría económica convencional. De acuerdo con la teoría prevaleciente, un aumento en el costo del crédito disminuye la competitividad internacional y, por ende, las exportaciones manufactureras. El signo positivo estaría indicando una relación directa entre la tasa de interés y las exportaciones; es decir, en Argentina, los aumentos recientes de la tasa de interés parecen provenir de un abultamiento de la demanda de crédito doméstico que, a su vez, estaría asociado con el proceso de expansión y diversificación de las exportaciones manufactureras. ${ }^{29}$ En síntesis, las decisiones de endeudamiento, inversión y producción de las empresas manufactureras podrían obedecer, en buena medida, a proyectos de exportación.

Por otra parte, la cIU reporta un coeficiente negativo y estadísticamente significativo a un nivel de $1 \%$. Esto sugiere que un aumento de la demanda interna (el cual se reflejaría en una mayor utilización de la capacidad instalada) traería consigo una disminución de los excedentes para

\footnotetext{
${ }^{28}$ Resulta hasta cierto punto paradójico que en México el parámetro de la IED no haya resultado estadísticamente significativo, puesto que este país ha sido tradicionalmente más exitoso que Argentina en la atracción de inversiones orientadas a la producción y exportación de manufacturas no basadas en recursos naturales, desde las de bajo hasta las de alto contenido tecnológico. Véase Mortimore et al. (2001: 25).

${ }^{29}$ Éste es un resultado atípico, para el cual no fue posible encontrar evidencia previa en el caso particular de Argentina. Sin embargo, empleando un modelo de ciclo real de negocios, King y Rebelo (2000: 38) seńalan que una mejora sostenida en la productividad puede estimular el crecimiento económico y, al mismo tiempo, elevar la tasa de interés real. Aun cuando estos autores no analizan el comportamiento de las exportaciones, sería razonable asumir que éstas aumentarían junto con la productividad y la oferta agregada. Por otra parte, analizando el caso de México, Cuevas (1999: 34) aporta evidencia empírica en el sentido de que un proceso de expansión económica puede coincidir con una trayectoria alcista de la tasa de interés real. En este marco, el incremento en la tasa real de interés obedecería a una elevación en la demanda de crédito interno.
} 
exportar y viceversa. Esta evidencia, sin embargo, es sensible al método econométrico utilizado en razón de que las funciones de impulso-respuesta para ambos países indican que una mayor CIU es consistente con una elevación de las exportaciones.

Finalmente, para asegurar la estabilidad de la ecuación y mejorar el comportamiento de los residuales, fue necesario incorporar una variable dicotómica $(D)$. Esta variable busca capturar los efectos de la crisis económica argentina de diciembre de 2001, así como el episodio de volatilidad cambiaria registrado a finales de 2007 y principios de $2008 .{ }^{30}$ Como puede verse, esta variable resultó estadísticamente significativa a un nivel de 5 por ciento. ${ }^{31}$

En el cuadro 6, acerca de la ecuación reespecificada de la economía mexicana, se puede observar que los parámetros de regresión asociados a la productividad laboral y a la demanda externa son positivos y estadísticamente significativos a un nivel de significancia de 5 y $1 \%$, respectivamente. ${ }^{32} \mathrm{Si}$ se considera que la primera diferencia de una variable constituye una proxy de su tasa de crecimiento, entonces podría decirse que un aumento de un punto porcentual en la tasa de crecimiento de la productividad laboral generaría un incremento de 76 puntos base (es decir, 76 centésimas de punto porcentual) en la tasa de crecimiento de las exportaciones manufactureras. De forma análoga, una baja de un punto porcentual en la tasa de crecimiento de los mercados foráneos provocaría un recorte de aproximadamente 49 puntos base en el ritmo de crecimiento de las exportaciones manufactureras. En este contexto, al igual que en el caso de Argentina, un paquete coherente de estímulos a la productividad laboral podría mitigar de manera tangible los efectos de una recesión internacional sobre las exportaciones manufactureras de México.

En otro orden de ideas, el parámetro vinculado al tipo de cambio real es estadísticamente significativo pero reporta un signo negativo, lo cual sugiere que una depreciación real de la moneda reduce las exportaciones; por lo menos, en un escenario de corto plazo. Como se dijo, esto podría deberse a que una depreciación real genera efectos de signo contrario sobre la competitividad internacional: por un lado, la fortalece mediante el abaratamiento de las exportaciones en términos de dólares y, por otro, la menoscaba a través del encarecimiento de los insumos y bienes de ca-

${ }^{30}$ De este modo, dicha variable asume el valor 1 en los cuatro trimestres de 2002 y en el primer trimestre de 2008, y cero en el resto del periodo.

${ }^{31}$ En lo sucesivo, salvo precisión en contrario, se entenderá que la significancia estadística se alcanza a un nivel de 5 por ciento.

${ }^{32}$ Esto es consistente con el hallazgo realizado para la economía argentina en el sentido de que tanto la tasa de crecimiento de la productividad del factor trabajo, como el ritmo de expansión de los mercados foráneos, han influido de manera importante en la trayectoria de las exportaciones manufactureras. 
pital importados en términos de pesos. El efecto neto parecería ser negativo, por lo menos, en un escenario de corto plazo. ${ }^{33}$ La prevalencia del efecto negativo podría deberse a la elevada dependencia de la economía mexicana respecto de los insumos intermedios y bienes de capital importados. Esta dependencia puede medirse mediante la participación de las importaciones de bienes intermedios y de capital en las importaciones totales. En el caso mexicano dicha participación alcanza $85.99 \%$, mientras que en el argentino se limita a $52.27 \%{ }^{34}$ Esto podría explicar, en alguna medida, que una depreciación real de la moneda incida de distinta manera en las exportaciones de manufacturas de una y otra nación.

En el cuadro 6 se observa, asimismo, que el coeficiente del personal ocupado sólo es estadísticamente significativo a un nivel de $10 \%$, pero la presencia de esta variable contribuye a mejorar los resultados de las pruebas de diagnóstico.

Finalmente, habría que señalar que los coeficientes de determinación ajustados (es decir, las $R^{2}$ ajustadas) para las regresiones de Argentina y México son iguales a 81.48 y $50.92 \%$, respectivamente. Considerando que se trata de regresiones en diferencias, el ajuste de curva podría calificarse como adecuado para Argentina y aceptable para México.

\subsection{Pruebas de diagnóstico}

Por lo que se refiere a las pruebas de diagnóstico, se procedió a descartar posibles problemas de autocorrelación, heteroscedasticidad, heteroscedasticidad autorregresiva condicional y anormalidad en los residuales de las regresiones reformuladas. Asimismo, se hicieron pruebas de estabilidad, las cuales llevaron a incluir la variable dicotómica en el caso de Argentina. En el cuadro 7 se reportan los resultados fundamentales para Argentina y México.

Como se puede constatar, los residuales de los modelos estimados tienen un comportamiento consistente con ruido blanco normal. Dicho de otro modo, los residuales de ambas regresiones se encuentran exentos de correlación serial y heteroscedasticidad, además de que siguen una distribución aproximadamente normal. ${ }^{35}$ Es importante destacar que,

\footnotetext{
${ }^{33}$ Este efecto negativo, sin embargo, podría eventualmente revertirse dependiendo de la rapidez y eficacia con la que pudieran sustituirse algunas importaciones, subsanarse cuellos de botella en distintos procesos productivos y forjarse nuevos canales de distribución para relanzar las exportaciones.

${ }^{34}$ Fuente: estimación propia con base en datos del Informe Anual del Banco de México 2009 (p. 134) y del Instituto Nacional de Estadística y Censos de la República Argentina. Es importante añadir que, si se excluyen las importaciones de bienes intermedios del sector petrolero de México, la medida de dependencia propuesta disminuye en menos de 5 puntos porcentuales, para situarse en 81.07 por ciento.

${ }^{35}$ Mediante las pruebas de correlación serial de Breusch-Godfrey se descartó no sólo la presencia de correlación serial de primer orden, sino también de orden superior.
} 


\section{Cuadro 7 \\ Pruebas de diagnóstico para las ecuaciones de regresión reespecificadas de Argentina y México}

\begin{tabular}{llcc}
\hline \multicolumn{1}{c}{ Tipo de prueba } & \multicolumn{1}{c}{ Hipótesis nula } & $\begin{array}{c}\text { Valor de } \\
\text { probabilidad para } \\
\text { Argentina }\end{array}$ & $\begin{array}{c}\text { Valor de } \\
\text { probabilidad } \\
\text { para México }\end{array}$ \\
\hline Correlación serial $^{\mathrm{a}}$ & $\begin{array}{l}\text { Ausencia de } \\
\text { correlación serial }\end{array}$ & 0.528077 & 0.228303 \\
Heteroscedasticidad $^{\mathrm{b}}$ & Homoscedasticidad & 0.855703 & 0.373032 \\
ARCH $^{\mathrm{c}}$ & Ausencia de ARCH & 0.808786 & 0.532424 \\
Normalidad $^{\mathrm{d}}$ & Normalidad & 0.309907 & 0.777784 \\
\hline
\end{tabular}

${ }^{a}$ Prueba Breusch-Godfrey de correlación serial para los residuales desde el primero hasta el quinto orden

${ }^{\mathrm{b}}$ Prueba de heteroscedasticidad de White para los residuales con dos rezagos y sin términos cruzados.

c Prueba de heteroscedasticidad autorregresiva condicional (prueba ARCH) para los residuales, empleando cinco rezagos.

${ }^{d}$ Prueba de normalidad Jarque-Bera.

Fuente: Elaboración propia.

para complementar la prueba de heteroscedasticidad de White, se llevó a cabo una prueba de heteroscedastidad autorregresiva condicional (o prueba ARCH) a diferentes rezagos, la cual descartó por completo la presencia de este fenómeno. Asimismo, se obtuvieron los correlogramas de los residuales de ambos modelos y se confirmó una vez más que su comportamiento es adecuado, a juzgar tanto por las funciones de autocorrelación y de autocorrelación parcial estimadas, como por las estadísticas $Q$ de Ljung y Box desde el primero hasta el vigésimo rezago. ${ }^{36}$

Por otra parte, se realizó la prueba de Chow con múltiples fechas de rompimiento estructural para ambos países. Como se señaló, en el caso particular de Argentina fue necesario incluir una variable dicotómica para lograr la estabilidad de la ecuación. Hecho esto, el resultado es que la hipótesis nula de ausencia de cambio estructural no puede rechazarse para país alguno. ${ }^{37}$

Finalmente, se utilizó una medida alternativa para la demanda externa de cada nación, con el objeto de verificar la solidez de la evidencia empírica obtenida. En el modelo que corresponde a la economía mexicana, en lugar de las exportaciones manufactureras totales de Estados Unidos, se empleó el PIB real de ese país (en primeras diferencias). ${ }^{38}$ En el cuadro BII del Apéndice II se confirma que, a un nivel de significancia

\footnotetext{
${ }^{36}$ Por razones de espacio, estos resultados están disponibles bajo pedido.

${ }^{37}$ Estos resultados se encuentran disponibles bajo pedido.

${ }^{38}$ Fuente: Bureau de Análisis Económico del Departamento de Comercio de Estados Unidos.
} 
de $10 \%$, los resultados que arroja esta nueva estimación son en esencia los mismos que se reportan en el cuadro 6 .

En el modelo sobre la economía argentina, en lugar de la variable originalmente propuesta para medir la demanda externa (las importaciones manufactureras totales realizadas por las 30 naciones de la OCDE y por los tres socios comerciales de Argentina en el marco del Mercosur), se usaron tres diferentes medidas de actividad económica externa: ${ }^{39}$ la producción industrial de la Unión Europea (UE), la producción industrial de Brasil, y el PIB real de Brasil. ${ }^{40}$ Aunque ninguna de estas medidas de demanda externa alcanza significancia estadística, los resultados para el resto de las variables del sistema no sufren modificaciones sustanciales. En el cuadro AII del Apéndice II se presentan los resultados obtenidos mediante el empleo del índice de producción industrial de Brasil (en primeras diferencias), pues esta variable fue la de mejor desempeño a juzgar por los valores de probabilidad, la capacidad explicativa del modelo y el comportamiento de los residuales. ${ }^{41} \mathrm{Si}$ se coteja el cuadro AI con el cuadro 5 , se confirma que los hallazgos en torno al efecto de la productividad laboral, la IED, el costo del crédito y la ciu sobre las exportaciones manufactureras argentinas, se mantienen vigentes a un nivel de significancia de 5 por ciento.

\section{Modelos GVAR estacionarios, pruebas multivariadas de diagnóstico y análisis de sensibilidad}

Como se vio, las variables seleccionadas para cada nación involucran diferentes órdenes de integración: en lo tocante a Argentina se concluyó que hay cuatro variables $\mathrm{I}(2)$ y cinco I(1), mientras que en lo concerniente a México hay ocho variables I(1) y una estacionaria (o I(0)). Por definición, las pruebas de cointegración de Johansen son útiles para determinar si un conjunto de variables no estacionarias, cuyo orden de integración es el mismo, guardan una relación a largo plazo. En este contexto, por una parte, se homogeneizó el orden de integración para las series de la economía argentina (es decir, las variables I(2) se expresaron en primeras dife-

\footnotetext{
${ }^{39}$ En el caso de Argentina, la búsqueda de medidas alternativas para la demanda externa estuvo limitada por la disponibilidad de series estadísticas completas con periodicidad trimestral.

${ }^{40}$ Fuente: la producción industrial de la uE se obtuvo de la base de datos de Eurostat, mientras que la producción industrial y el PIB real de Brasil se obtuvieron del Instituto Brasileño de Geográfica y Estadística.

${ }^{41}$ En 2009, 18.8\% de las exportaciones argentinas se colocaron en Brasil, lo cual convierte a este país en el principal destino de exportación para Argentina (Base de Datos de Comercio Exterior de Argentina). Asimismo, las exportaciones argentinas a la uE representaron $18.7 \%$ del total en 2008 , lo cual la convierte en el principal socio comercial de Argentina después de Brasil (Delegación de la Comisión Europea en Argentina, 2009, http://www.delarg.ec.europa.eu/es/novedades/documentos/ brochure2.pdf).
} 
rencias) y, por la otra, se excluyó la variable estacionaria de la economía mexicana (es decir, se excluyó la IED). Bajo la premisa de que todas las variables involucradas eran $\mathrm{I}(1)$, se realizaron pruebas de cointegración de Johansen para cada país pero no fue posible identificar uno solo de los vectores de cointegración resultantes, puesto que los signos de los parámetros a largo plazo se alejan demasiado de lo establecido por la teoría económica. En este contexto, el análisis de sensibilidad se realiza con base en sendos modelos VAR estacionarios y funciones generalizadas de impulso-respuesta. En virtud de que no se identificaron relaciones de cointegración, el punto de partida es el siguiente modelo vaR no restringido.

$$
Y_{t}=\mathrm{B}_{0}+\sum_{i=1}^{p} \mathrm{~B}_{i} Y_{t-i}+\varepsilon_{t}
$$

donde $Y_{t}$ es un vector de variables de dimensión $(9 \times 1)$, $\mathrm{B}_{0}$ es un vector de términos constantes de dimensión $(9 \times 1), \mathrm{B}_{i}$ es la iésima matriz de coeficientes de dimensión $(9 \times 9)$, donde $i=1,2, \ldots, p$, y $\varepsilon_{t}$ es un vector de innovaciones de dimensión $(9 \times 1)$. A reserva de hacer las pruebas de diagnóstico respectivas, aquí se parte de los siguientes supuestos básicos:

1) Todas las raíces invertidas del polinomio autorregresivo del modelo var (es decir, las raíces de $\left|I_{9}-\sum_{i=1}^{P} \mathrm{~B}_{i} L^{i}\right|=0$, donde $\mathrm{L}$ es el operador de rezago) residen dentro del círculo unitario. De allí que el modelo sea estable.

2) Las innovaciones siguen una distribución normal multivariada con media cero y varianza constante. Formalmente, $\varepsilon_{t}-N_{9}(0, \Sigma)$, donde $\sum$ es la matriz de covarianzas del vector innovaciones; es decir, $E\left(\varepsilon_{t} \varepsilon_{t}\right)=\Sigma$. En virtud de que las varianzas se encuentran en la diagonal principal y son todas positivas, $\Sigma$ es una matriz definida positiva.

3) Las innovaciones se encuentran exentas de correlación serial. Formalmente, $E\left(\varepsilon_{t} \varepsilon_{t}\right)=0$ para toda $t \neq s$.

\subsection{Metodología VAR generalizada}

Normalmente, los elementos del vector de innovaciones $\left(\varepsilon_{t}\right)$ se correlacionan entre sí, por lo que $\Sigma=\left\{\sigma_{i j} \mathrm{i}, \mathrm{j}=1,2, \ldots, 9\right\}$ es una matriz no diagonal. La correlación contemporánea entre los elementos de $\varepsilon_{t}$ ocasiona que no se puedan atribuir innovaciones a variables específicas. Dicho de otro modo, en principio no se puede hablar de innovaciones a la productividad laboral, al tipo de cambio real o a la demanda externa de exportaciones. Es importante subrayar que en este trabajo se recurre al método 
de Pesaran y Shin (1998) para eliminar la correlación contemporánea y estimar funciones generalizadas de impulso-respuesta (FGIR), cuya principal ventaja es que no dependen de la ordenación de las ecuaciones del modelo. ${ }^{42}$ Para explicar la diferencia entre esta metodología y la tradicional, es conveniente asumir que nuestro modelo var es de primer orden $(\operatorname{VAR}(1))$ :

$$
Y_{t}=\mathrm{B}_{0}+\mathrm{B}_{1} Y_{t-1}+\varepsilon_{t}
$$

Enseguida se recurre al primer supuesto, el cual implica que el vector $Y_{t}$ es estacionario en covarianza, para reexpresar el modelo vAR como un vector de promedio móvil de orden infinito.

$$
Y_{t}=\mu+\sum_{i=0}^{\infty} \mathrm{B}_{1}^{i} \varepsilon_{t-i}
$$

donde $\mu=\left(I_{9}-A_{1}\right)^{-1} \mathrm{~B}_{0}$ y representa la media del proceso. Bajo el enfoque tradicional, propuesto por Sims (1980), el problema de la correlación contemporánea entre las innovaciones se resuelve mediante la descomposición de Cholesky de la matriz $\Sigma$. Es decir, dado que $\sum$ es una matriz definida positiva (supuesto 2 ), simétrica y no diagonal, existe una matriz triangular inferior $L$ tal que:

$$
\Sigma=L L^{\prime}
$$

En este contexto, si premultiplicamos $\varepsilon_{t}$ por la inversa de $L$, entonces se obtiene el vector de innovaciones ortogonalizadas: $L^{-1} \varepsilon_{t}=u_{t}$. La ecuación (6) demuestra que la matriz de covarianzas del vector $u_{t}$ es una matriz identidad de dimensión $(9 \times 9)$, lo cual significa que los elementos de dicho vector están exentos de correlación contemporánea y han sido normalizados para tener una varianza igual a uno.

$$
E\left(u_{t} u_{t}\right)=E\left(L^{-1} \varepsilon_{t} \varepsilon_{t}^{\prime} L^{-1}\right)=L^{-1} E\left(\varepsilon_{t} \varepsilon_{t}\right) L^{-1}{ }^{\prime}=L^{-1} \sum L^{-1}{ }^{\prime}=L^{-1} L L^{\prime} L^{-1}{ }^{\prime}=I_{9}
$$

Enseguida se procede a reexpresar la ecuación (3) en términos del vector de innovaciones ortogonalizadas:

$$
Y_{t}=\mu+\sum_{i=0}^{\infty}\left(\mathrm{B}_{1}^{i} L\right) L^{-1} \varepsilon_{t-i}=\mu+\sum_{i=0}^{\infty}\left(\mathrm{B}_{1}^{i} L\right) u_{t-i}
$$

Finalmente, la ecuación (8) representa al vector de funciones ortogonalizadas de impulso-respuesta $\left(\psi^{\circ}\right)$. En este caso, el impulso o choque

${ }^{42}$ A diferencia de los modelos var recursivos, los modelos GVAR producen evidencia empírica que no depende del orden en que se coloquen las variables involucradas. 
se produce sobre ecuación $j$ del vector $Y_{t}$; es decir, el impulso tiene lugar en el periodo $t$ y se observa la respuesta en el periodo $t+n$.

$$
\psi_{j}^{\rho}(n)=\mathrm{B}^{n} L e_{j}
$$

donde $\mathrm{n}=0,1,2, \ldots$, y $e_{j}$ es un vector hipotético de innovaciones de dimensión $(9 \times 1)$, el cual contiene un uno en la hilera $j$ y ceros en el resto de las hileras. Esta forma de identificar funciones de impulso-respuesta (apoyada en una descomposición triangular de $\Sigma$ ) presenta el problema de que es recursiva. Dicho de otro modo, da lugar a una estructura asimétrica en la que un choque a una determinada variable surte efectos contemporáneos en las variables que le siguen en la ordenación de las ecuaciones, mientras que las variables que le anteceden se verán afectadas sólo de manera desfasada; es decir, a través de la estructura de rezagos del modelo VAR. ${ }^{43}$

Con el objeto de obtener evidencia empírica que no dependa de la ordenación de las ecuaciones, Pesaran y Shin (1998) desarrollan un método para estimar funciones generalizadas de impulso-respuesta (FGIR). La idea central es que una función de impulso-respuesta está dada por la diferencia entre el valor esperado de una variable en el periodo $t+n$, después de ocurrido un choque en el periodo $t$, y su valor esperado en el periodo $t+n$ en ausencia del choque referido. Este segundo valor se obtiene a partir del comportamiento histórico de la economía. Por ejemplo, si se produce un choque de magnitud $\delta$ sobre la ecuación $j$ del vector $Y$, entonces el vector de FGIR estaría representado por:

$$
F G I R_{y}\left(n, \delta, \Omega_{t-1}\right)=E\left(Y_{t+n} \mid \varepsilon_{j t}=\delta, \Omega_{t-1}\right)-E\left(Y_{t+n} \mid \Omega_{t-1}\right)
$$

donde la matriz $\Omega_{t-1}$ representa la información disponible sobre el comportamiento histórico de la economía hasta el periodo $t$ - 1 . Apelando al segundo supuesto de que las innovaciones $(\varepsilon)$ siguen una distribución normal multivariada, puede inferirse con base en los trabajos tanto de Pesaran y Shin (1998) como de Koop et al. (1996) que:

$$
E\left(\varepsilon_{t} \mid \varepsilon_{j t}=\delta\right)=\left(\sigma_{1 j}, \sigma_{2 j}, \ldots, \sigma_{9 j}\right)^{\prime} \sigma_{j j}^{-1} \delta=\sum e_{j} \sigma_{j j}^{-1} \delta
$$

Luego entonces, en este caso, el vector de FGIR no normalizadas estaría dado por:

\footnotetext{
${ }^{43}$ Formalmente, una innovación o choque a la variable $Y_{j t}$ tendrá un efecto contemporáneo en la variable $Y_{i t}$ sí y sólo sí $i \geq j$.
} 


$$
\left.\left(\frac{\mathrm{B}^{n} \Sigma e_{j}}{\sqrt{\sigma_{j j}}}\right) \frac{\delta}{\sqrt{\sigma_{j j}}}\right)
$$

Finalmente, para obtener el vector de FGIR normalizadas simplemente igualamos $\delta$ a $\sqrt{\sigma_{j j}}$. Esto es,

$$
\psi_{j}^{G}(n)=\left(\frac{\mathrm{B}^{n} \Sigma e_{j}}{\sqrt{\sigma_{j j}}}\right)
$$

Vale señalar que $\psi_{j}^{G}(n)$ mide el efecto de un choque de una desviación estándar en la ecuación $j$. El choque tiene lugar en el periodo $t$ y repercute en los valores esperados del vector $Y$ en el periodo $t+n$, donde $n=$ $0,1,2, \ldots$

\subsection{Desarrollo empírico}

Para la adecuada estimación de los modelos GVAR correspondientes a México y Argentina, en primer lugar se probaron diferentes especificaciones para la estructura de rezagos y el espacio de información (o selección de variables). En cada caso, se buscó un equilibrio razonable entre la relevancia teórica, el adecuado comportamiento de residuales, y eficiencia en la estimación de parámetros. Con respecto a la estructura de rezagos, después de recurrir a cinco criterios automáticos, se optó por utilizar un rezago para cada variable en cada ecuación, tanto en el caso argentino como en el mexicano. Cabe seńalar que algunos criterios penalizan en mayor o menor grado la inclusión de rezagos adicionales y tienden, por ende, a discrepar en sus resultados. De allí que hubiera que decidir con base en el comportamiento de los residuales y en la pertinencia de dejar suficientes grados de libertad para estimar eficientemente los parámetros de cada modelo. ${ }^{44}$

En segundo lugar, después de estudiar las relaciones de intercambio entre la estructura de rezagos y el espacio de información, se consideró conveniente excluir la IED y la tasa de interés en ambos modelos. Asimismo, se eliminó el personal ocupado (Po) en el caso de Argentina y los salarios $(W)$ en el de México. En síntesis, se suprimieron tres variables en cada modelo. Dos razones justifican esta decisión: la primera es que las variables referidas no generan funciones de impulso-respuesta que sean estadísticamente significativas, mientras que la segunda es que la inclusión de cualquiera de ellas redunda en problemas de correlación serial y/o

\footnotetext{
${ }^{44}$ La decisión tomada es consistente con los resultados de los criterios basados, por una parte, en el error final de predicción y, por la otra, en el cociente de verosimilitud secuencial modificado.
} 
anormalidad en los residuales, que no fue posible corregir mediante un mayor número de rezagos o por medio de la inclusión de variables indicadoras. ${ }^{45}$

De esta manera, el modelo empleado para Argentina quedaría representado por: $Y_{t}=\left[\Delta^{2} X, \Delta v_{t}, \Delta^{2} W_{t}, \Delta Q, \Delta D E_{t} \Delta^{2} C I U_{t}\right]^{\prime}$. Puesto que los modelos deben ser estacionarios, en el caso de Argentina algunas variables tuvieron que expresarse en segundas diferencias. Asimismo, la especificación correspondiente a México sería: $Y_{t}=\left[\Delta X_{t}, \Delta v_{t}, \Delta Q, \Delta D E_{t}, \Delta P O_{t}\right]^{\prime}$. Para cada nación, como se puede ver, se tendría un modelo GVAR consistente de seis ecuaciones.

\subsubsection{Pruebas multivariadas de diagnóstico}

Para demostrar la congruencia de estas especificaciones, enseguida se presentan los resultados de las pruebas de correlación serial de BreuschGodfrey, desde el primero hasta el quinto orden (o quinto trimestre). Como se constata en el cuadro 8 , todos los valores de probabilidad asociados a la hipótesis nula de ausencia de correlación serial son muy superiores a 5\%, tanto para Argentina como para México. Esto significa que, a un nivel de significancia de 5\%, la hipótesis nula de ausencia de correlación serial no puede rechazarse en caso alguno. ${ }^{46}$

\section{Cuadro 8}

\section{Pruebas de correlación serial de Breusch-Godfrey $H_{0}$ : no existe correlación serial hasta el rezago $p$}

\begin{tabular}{|c|c|c|}
\hline Rezago (p) & $\begin{array}{l}\text { Valores de probabilidad } \\
\text { para Argentina }\end{array}$ & $\begin{array}{c}\text { Valores de probabilidad } \\
\text { para México }\end{array}$ \\
\hline 1 & 0.4065 & 0.5375 \\
\hline 2 & 0.3664 & 0.1198 \\
\hline 3 & 0.2559 & 0.5255 \\
\hline 4 & 0.3219 & 0.7213 \\
\hline 5 & 0.3942 & 0.3503 \\
\hline
\end{tabular}

Nota: Probabilidades asociadas con una distribución $X^{2}$ con 36 grados de libertad. Fuente: Elaboración propia.

El cuadro 9, por su parte, muestra que la versión multivariada de la prueba de heteroscedasticidad de White no permite rechazar la hipótesis nula de homoscedasticidad para país alguno, ni siquiera a un nivel de signi-

\footnotetext{
pedido.

${ }^{46}$ De hecho, la hipótesis de referencia no se rechaza ni siquiera a un nivel de significancia de 10 por ciento.
}

${ }^{45}$ Estos resultados no aparecen por razones de espacio, pero se encuentran disponibles bajo 
ficancia de $10 \% \cdot{ }^{47}$ Finalmente, en el cuadro 10 se aprecia que los residuales de ambos modelos siguen una distribución aproximadamente normal, en razón de que la hipótesis nula de normalidad no puede rechazarse para país alguno, ni siquiera a un nivel de significancia de 10 por ciento.

\section{Cuadro 9}

Prueba multivariada de heteroscedasticidad de White $H_{0}$ : homoscedasticidad

\begin{tabular}{cc}
\hline Valor de probabilidad para Argentina & Valor de probabilidad para México \\
\hline 0.2738 & 0.3933 \\
\hline
\end{tabular}

Notas: Se incluyen términos cruzados en las ecuaciones de prueba de cada nación. Probabilidades asociadas con una distribución $\chi^{2}$ con 567 grados de libertad.

Fuente: Elaboración propia.

\section{Cuadro 10 \\ Prueba multivariada de normalidad Jarque-Bera $H_{0}$ : los residuales siguen una distribución normal multivariada}

\begin{tabular}{cc}
\hline Valor de probabilidad para Argentina & Valor de probabilidad para México \\
\hline 0.9164 & 0.4365 \\
\hline
\end{tabular}

Nota: Las pruebas de normalidad están basadas en el método de Lütkepohl, el cual se apoya en la ortogonalización de Cholesky.

Fuente: Elaboración propia.

No obstante que todas las variables son estacionarias, se verificó que ambos modelos fueran estables; es decir, se corroboró que en cada caso las raíces invertidas del polinomio autorregresivo característico tuvieran un módulo inferior a uno y residieran dentro del círculo unitario. La estabilidad es condición suficiente para la estacionariedad del sistema en su conjunto y garantiza que los efectos acumulados de los choques sean calculables y finitos. ${ }^{48}$

\subsubsection{Análisis de sensibilidad}

Enseguida se estima la respuesta dinámica de las exportaciones manufactureras de cada país frente a incrementos en algunos de sus determinantes básicos: la productividad laboral, los salarios, el tipo de cambio real y la demanda externa de exportaciones. Esto se hace mediante funciones

\footnotetext{
${ }^{47}$ Por razones de espacio, sólo se reportan los resultados de la prueba conjunta de heteroscedasticidad. Los resultados de las pruebas para los componentes individuales también descartan la presencia de heteroscedasticidad y se encuentran disponibles bajo pedido.

${ }^{48}$ Los resultados de las pruebas de estabilidad se encuentran disponibles.
} 
generalizadas de impulso-respuesta (FGIR), las cuales se estimaron en horizontes de 12 meses y con intervalos de aproximadamente $95 \%$ de confianza. Como se señaló, las FGIR no dependen de la ordenación de las ecuaciones del modelo. Es conveniente destacar que, en cada caso, lo que se observa es cómo responden las exportaciones de manufacturas frente a choques en diferentes variables. Los choques, en este contexto, se definen como incrementos no anticipados cuya duración es de un trimestre (es decir, se trata de choques temporales) y cuya magnitud equivale a una desviación estándar. La respuesta frente a los choques se ilustra mediante las FGIR y los intervalos respectivos son útiles para establecer significancia estadística. Es decir, una FGIR es estadísticamente significativa (o diferente de cero) cuando su intervalo de confianza excluye al cero en algún punto dentro del horizonte de 12 meses. La figura I ilustra las relaciones dinámicas correspondientes a la economía argentina.

En esta figura se observa que un choque a la productividad laboral incrementa las exportaciones manufactureras en el momento del impacto y el efecto se diluye poco después; sintomático de esto es que el intervalo de confianza respectivo comience a incluir al cero. Como se puede apreciar, una expansión de la demanda externa genera un efecto similar, aunque ligeramente más notorio y persistente, sobre las exportaciones. Las depreciaciones reales de la moneda y los ajustes salariales no producen una respuesta significativa de las exportaciones.

Por otra parte, es interesante destacar que los choques a la capacidad instalada utilizada (CIU) aumentan las exportaciones en lugar disminuirlas. Esto es consistente con la hipótesis de autoselección, según la cual un aumento de la ciu obedecería a una decisión consciente de la empresa con miras a la exportación. Diversas investigaciones empíricas indican que las decisiones en materia de producción e inversión de las empresas exportadoras podrían estar respondiendo, sobre todo, a proyectos de exportación. ${ }^{49}$

Finalmente, el hecho de que un choque exportador (o choque propio) estimule de manera sustantiva las exportaciones de manufacturas sugiere que el comportamiento de esta variable involucra un fuerte componente predeterminado o inercial debido, posiblemente, a que las relaciones comerciales tardan algún tiempo no sólo en forjarse, sino también en finiquitarse. En lo referente a México, como se puede constatar, los hallazgos que se realizan son muy similares (figura II).

${ }^{49}$ Álvarez y López (2005) aportan evidencia sólida de que las empresas chilenas se autoseleccionan de manera consciente para penetrar los mercados internacionales, por lo que muchas decisiones estratégicas podrían estar orientadas precisamente en ese sentido. Asimismo, Wagner (2007), Girma et al. (2004) y Hallward-Driemeier et al. (2002), entre otros, proporcionan evidencia para muchas otras naciones que refuerza la noción de que las empresas se autoseleccionan para exportar y, por ende, toman decisiones y canalizan recursos en esa dirección. 


\section{Figura I}

Respuesta de las exportaciones manufactureras argentinas frente a choques temporales en diferentes variables
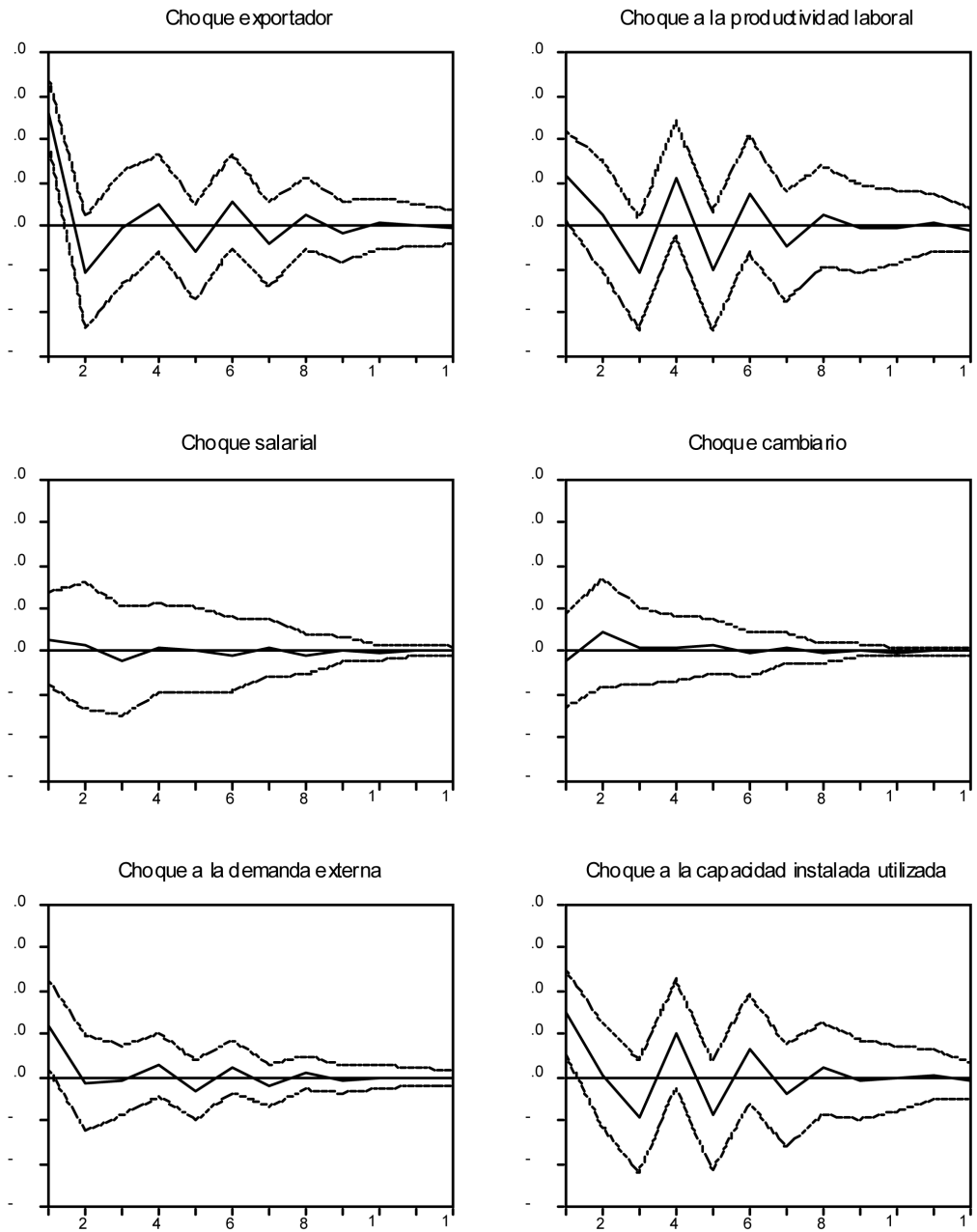

Fuente: Elaboración propia.

En México, tanto las mejoras en la productividad de los trabajadores como los aumentos en la demanda externa, se traducen en mayores exportaciones manufactureras. En ambos casos, los efectos positivos se producen en el momento del impacto y tardan aproximadamente un mes en disiparse. Si se considera que los choques a la productividad laboral y a la demanda externa son meramente transitorios, entonces podría concluirse que las exportaciones manufactureras son sensibles frente a cambios 


\section{Figura II}

Respuesta de las exportaciones manufactureras mexicanas frente a choques temporales en diferentes variables
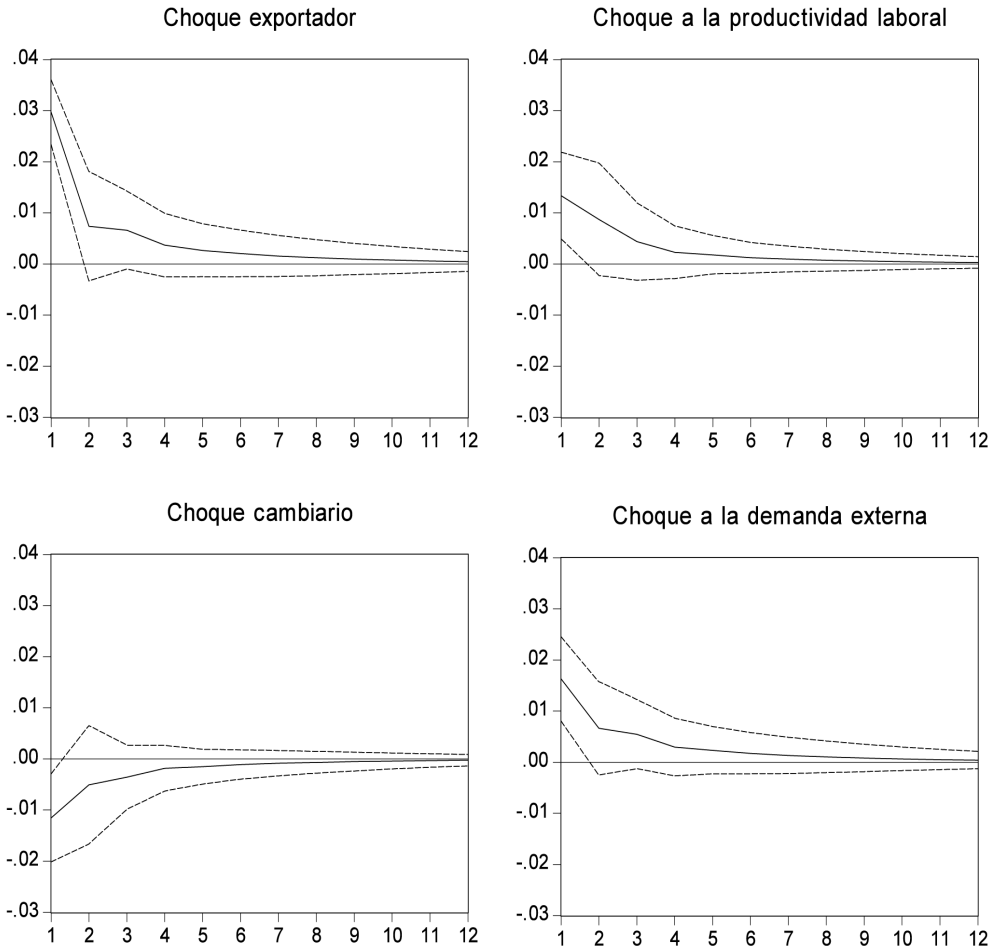

Choque a la capacidad instalada utilizada

Choque al personal ocupado
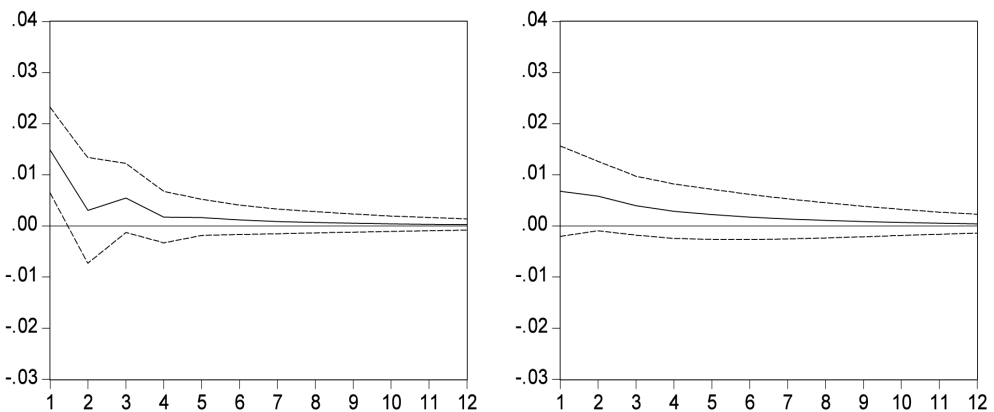

Fuente: Elaboración propia. 
en estas variables. Las FGIR alcanzan mayor significancia estadística en México que en Argentina, lo cual muy probablemente se debe a que las exportaciones manufactureras en el primer país se expresan en primeras diferencias, mientras que en el segundo tuvieron que diferenciarse dos veces para alcanzar estacionariedad. ${ }^{50}$

En otro orden de ideas, una depreciación real de la moneda disminuye (en lugar de acrecentar) las exportaciones manufactureras. Esto es consistente con lo observado en el análisis de regresión y, como se ha explicado suficientemente, podría ser el resultado de una pérdida temporal de competitividad internacional asociada al encarecimiento de los insumos y bienes de capital importados. Finalmente, en México la respuesta de las exportaciones frente a un choque exportador (o innovación propia) vuelve a exhibir un marcado componente inercial o predeterminado. Los choques a la ciu reportan de nueva cuenta un efecto positivo y estadísticamente significativo, lo cual refuerza la hipótesis de que las decisiones de producción e inversión de las empresas manufactureras obedecen más a sus planes y proyectos de exportación que a la evolución de la demanda interna.

Finalmente, aun cuando en algunos casos los intervalos de confianza sugieren que las FGIR son estadísticamente significativas, debe admitirse que los efectos de los choques se diluyen rápidamente. Esto se debe no sólo a la estructura de rezagos empleada, sino a que los choques duran sólo un trimestre y su magnitud es de una desviación estándar. Presumiblemente, si los choques fueran más prolongados o de mayor magnitud, los efectos referidos tardarían más en disiparse.

\section{Conclusiones}

En este trabajo se emplean dos métodos econométricos para estudiar los determinantes de las exportaciones manufactureras en Argentina y México. La evidencia empírica obtenida es consistente al indicar que, en ambas naciones, las exportaciones manufactureras responden positivamente frente a incrementos en la productividad laboral y la demanda externa. Tanto las elasticidades estimadas como las funciones de impulso-respuesta sugieren que la productividad de los trabajadores es una variable clave para estimular las exportaciones manufactureras. Una importante implicación de política económica es que, con medidas de aliento a la productividad del factor trabajo, es factible contrarrestar los

\footnotetext{
${ }^{50}$ Sims (1980) y Doan (2000), entre otros, plantean que la diferenciación provoca pérdida de información en los modelos VAR, aun cuando las series involucradas sean no estacionarias. En el caso de Argentina, la pérdida potencial de información con respecto a los comovimientos de las series sería mayor puesto que, en algunas instancias, fue necesario obtener segundas diferencias.
} 
efectos negativos de una contracción de la demanda externa sobre las exportaciones manufactureras. Esto significa que, en una coyuntura de recesión internacional, una mayor inversión en capacitación y adiestramiento de los trabajadores podría contribuir a mantener el potencial exportador de la industria referida.

Los efectos de otras variables sobre las exportaciones del sector pueden variar dependiendo de la técnica econométrica utilizada y/o del país analizado. Los efectos del tipo de cambio real sobre las exportaciones de manufacturas varían de un país a otro, pero no de una metodología econométrica a otra. En el caso de México, tanto las elasticidades estimadas como las funciones de impulso-respuesta son indicativas de que una depreciación real de la moneda reduce las exportaciones manufactureras. Esto parece obedecer a que la depreciación real genera efectos de signo contrario sobre la competitividad internacional: por una parte, la fortalece por medio del abaratamiento de las exportaciones en términos de dólares y, por otra, la debilita con el encarecimiento de insumos y bienes de capital importados en términos de pesos. En el corto plazo, parecería que el segundo efecto es dominante en la economía mexicana. En el caso de Argentina, por otro lado, el tipo de cambio real no parece influir en las exportaciones manufactureras; lo cual sugiere que, al menos en el corto plazo, los efectos positivo y negativo de una depreciación real sobre el volumen exportado se cancelan mutuamente. En esta tesitura, un programa amplio y coherente de estímulo a la productividad laboral sería más eficaz que nuevas depreciaciones reales de la moneda, en lo referente al aumento y la diversificación de las exportaciones.

Asimismo, la evidencia empírica es consistente con la hipótesis de autoselección, al sugerir que las decisiones de endeudamiento, inversión, producción y contratación de personal de las empresas obedecen en alguna medida a proyectos de exportación. Sintomático de lo anterior es que: 1) una elevación de la ciu aumente las exportaciones en ambas naciones, según las funciones de impulso-respuesta; 2) la tasa de interés guarde una relación positiva y estadísticamente significativa con las exportaciones en Argentina, como se desprende del cuadro 5; y 3) el personal ocupado exhiba una relación positiva y estadísticamente diferente de cero (a un nivel de significancia de 10\%) con las exportaciones en México, como se observa en el cuadro 6. Finalmente, el análisis de regresión alcanza a revelar el papel de la IED como factor de impulso a las exportaciones, pero este hallazgo es exclusivo de la economía argentina y se desvanece al transitar de un método econométrico a otro. 


\section{Apéndice I}

\section{Figura AI}

\section{Comportamiento de las variables I(2) en Argentina}
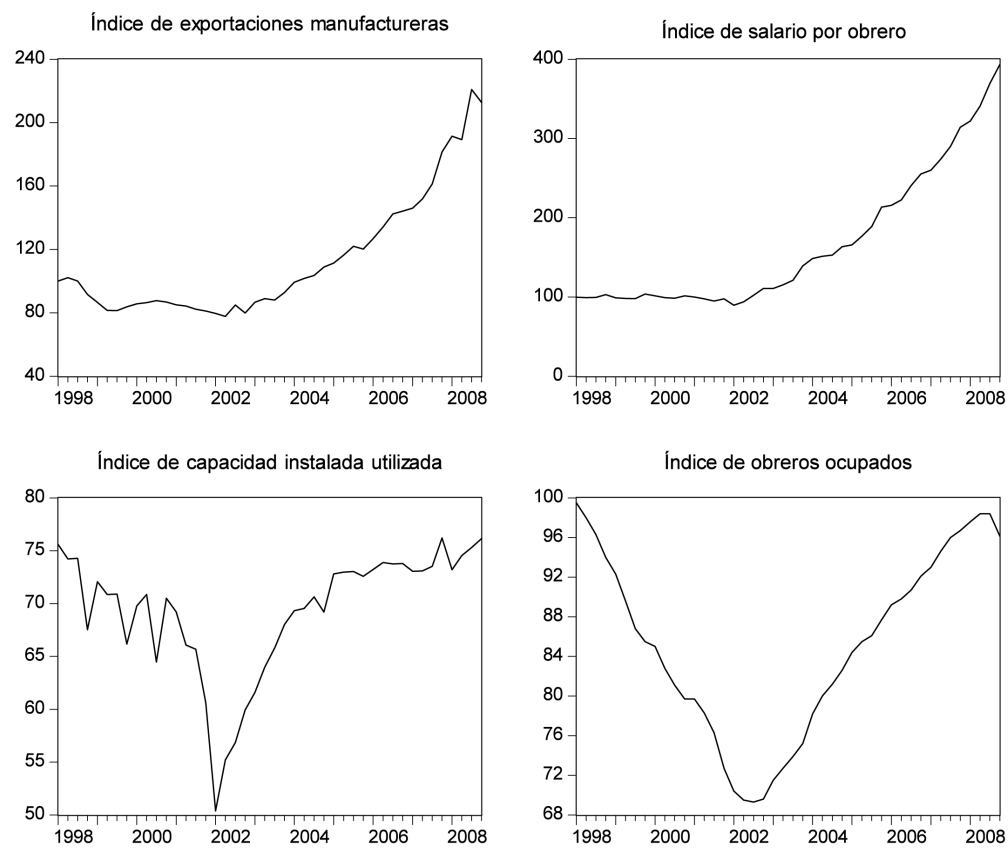

Fuente: Elaboración propia. 


\section{Apéndice II}

\section{Cuadro AII}

Ecuación reespecificada de las exportaciones manufactureras correspondiente a Argentina, empleando la producción industrial de Brasil como variable proxy para la demanda externa

\begin{tabular}{|c|c|c|c|c|}
\hline \multicolumn{5}{|c|}{ Variable dependiente: $\Delta^{2} X_{t}$} \\
\hline Regresores & Coeficiente & Error estándar & Estadistica $t$ & $\begin{array}{c}\text { Valor de } \\
\text { probabilidad }\end{array}$ \\
\hline$\Delta v_{\mathrm{t}}$ & 1.956567 & 0.429698 & 4.553354 & 0.0003 \\
\hline$\Delta D E_{t}$ & -0.091329 & 0.107139 & -0.852435 & 0.4058 \\
\hline$\Delta \mathrm{IED}_{t}$ & 0.000113 & 4.12E-05 & 2.748420 & 0.0137 \\
\hline$\Delta R_{t}$ & 0.002223 & 0.000641 & 3.467212 & 0.0029 \\
\hline$\Delta^{2} C I U_{t}$ & -0.726446 & 0.251724 & -2.885878 & 0.0103 \\
\hline$D_{t}$ & -0.042233 & 0.023936 & -1.764403 & 0.0956 \\
\hline Intercepto & -0.015943 & 0.009402 & -1.695612 & 0.1082 \\
\hline$R^{2}$ ajustada & 0.717723 & \multicolumn{2}{|c|}{ Error estándar de la regresión } & 0.033483 \\
\hline Estadística DW & 1.957925 & \multicolumn{2}{|c|}{ Valor de prob. de la prueba $F$} & 0.000054 \\
\hline
\end{tabular}

Nota: $D_{t}$ es una variable dicotómica para capturar los efectos de la crisis económica argentina de diciembre de 2001, así como el episodio de volatilidad cambiaria de finales de 2007 y principios de 2008. De allí que dicha variable asuma el valor 1 en los tres primeros trimestres de 2002 y en el primer trimestre de 2008, y cero en el resto del periodo.

Fuente: Elaboración propia.

\section{Cuadro BII}

Ecuación reespecificada de las exportaciones manufactureras correspondiente a México, empleando el PIB real de Estados Unidos como variable proxy para la demanda externa

Variable dependiente: $\Delta X_{t}$

\begin{tabular}{lrrrr}
\hline \multicolumn{1}{c}{ Regresores } & Coeficiente & Error estándar & Estadísticat & $\begin{array}{c}\text { Valor de } \\
\text { probabilidad }\end{array}$ \\
\hline$\Delta v_{\mathrm{t}}$ & 0.689190 & 0.379415 & 1.816455 & 0.0764 \\
$\Delta Q_{t}$ & -0.177067 & 0.084135 & -2.104553 & 0.0414 \\
$\Delta D E_{t}$ & 1.110443 & 0.637797 & 1.741061 & 0.0890 \\
$\Delta P O_{t}$ & 1.345143 & 0.466551 & 2.883166 & 0.0062 \\
Intercepto & 0.004791 & 0.005901 & 0.811988 & 0.4214 \\
$R^{2}$ ajustada & 0.438908 & Error estándar de la regresión & 0.023903 \\
Estadística DW & 2.358768 & Valor de prob. de la prueba $F$ & 0.000055 \\
\hline
\end{tabular}

Fuente: Elaboración propia. 


\section{Bibliografía}

Álvarez, Roberto y Ricardo López (2005), "Exporting and performance: evidence from Chilean plants", Canadian Journal of Economics, 38 (4), Canadian Economics Association, Montreal, pp. 1384-1400.

Berrettoni, Daniel y Sebastián Castresana (2007), "Exportaciones y tipo de cambio real: el caso de las manufacturas industriales argentinas", Revista de Comercio Exterior e Integración, 9, Centro de Economía Internacional, Buenos Aires, pp. 101-117.

Catao, Luis y Elisabetta Falsetti (2002), "Determinants of Argentina's external trade", Journal of Applied Economics, 5 (1), Universidad del cema, Buenos Aires, pp. 19-57.

Cuevas, Víctor (1999), "Efectos de la liquidez y de las expectativas inflacionarias de un choque monetario: un análisis de sensibilidad para el caso de México", Economía, Teoría y Práctica, 11, Universidad Autónoma Metropolitana, México, pp. 25-46.

Cuevas, Víctor (2008), "Efectos de la productividad laboral en las exportaciones manufactureras mexicanas", Comercio Exterior, 58 (6), Banco Nacional de Comercio Exterior, México, pp. 465-479.

Delegación de la Comisión Europea en Argentina (2009), <http://www. delarg.ec.europa.eu/es/novedades/documentos/brochure2.pdf>, 9 de mayo de 2009.

Dickey, David y Wayne Fuller (1981), "Likelihood ratio statistics for autoregressive time series with a unit root", Econometrica, 49, The Econometric Society, Cleveland, Ohio, pp. 1057-1072.

Dickey, David, William Bell y Robert Miller (1986), "Unit roots in time series models: tests and implications", The American Statistician, 40, American Statistical Association, Alexandria, Virginia, pp. $12-26$.

Doan, Thomas (2000), RATS user's manual, Version 5, Estima.

Garcés, Daniel (2008), "Análisis de las funciones de importación y exportación de México (1980-2000)”, El Trimestre Económico, 75 (1), Fondo de Cultura Económica, México, pp. 109-141. 
Girma, Sourafel, David Greenaway y Richard Kneller (2004), "Does exporting increase productivity? A microeconomic analysis of marched firms", Review of International Economics, 12 (5), International Economics and Finance Society, Pittsburgh, pp. 855-866.

Goldberg, Linda y Michael Klein (1997), "Foreign direct investment, trade and real exchange rate linkages in Southeast Asia and Latin America”, Documento de Trabajo núm. 6344, National Bureau of Economic Research, Cambridge, Massachusetts.

Greene, William (2008), Econometric Analysis, Pearson-Prentice Hall, Nueva Jersey.

Gujarati, Damodar (2004), Econometría, McGraw Hill, México.

Hallward-Driemeier, Mary, Giuseppe Iarossi y Kenneth Sokoloff (2002), "Exports and manufacturing productivity in East Asia: a comparative analysis with firm-level data", Documento de Trabajo núm. 8894, National Bureau of Economic Research, Cambridge, Massachusetts.

Hamilton, James (1994), Time series analysis, Princeton University Press, Princeton.

Jiménez, Félix, Giovanna Aguilar y Javier Kapsoli (1998), "Competitividad en la industria manufacturera peruana", <http://www.pucp. edu.pe/departamento/economia/images/documentos/DDD148. pdf>, 6 de mayo 2009.

King, Robert y Sergio Rebelo (2000), "Resuscitating real business cycles", Documento de Trabajo núm. 7534, National Bureau of Economic Research, Cambridge, Massachusetts.

Koop, Gary, Hashem Pesaran y Simon Potter (1996), "Impulse response analysis in nonlinear multivariate models", Journal of Econometrics, 74 (1), Elsevier, Ámsterdam, pp. 119-147.

Kwiatkowski, Denis, Peter Phillips, Peter Schmidt y Yoncheol Shin (1992), "Testing the null hypothesis of stationary against the alternative of a unit root," Journal of Econometrics, 54, John Wiley \& Sons, Los Ángeles, pp. 159-178. 
Loría-Díaz, Eduardo (2001), "La restricción externa dinámica al crecimiento de México a través de las propensiones del comercio, 1970-1999”, Estudios Económicos, 16 (2), El Colegio de México, México, pp. 227-251.

MacKinnon, James (1996), "Numerical distribution functions for unit root and cointegration tests", Journal of Applied Econometrics, 11, John Wiley \& Sons, Los Ángeles, pp. 601-618.

Mbaye, Ahmadou y Stephen Golub (2002), "Unit labour cost, international competitiveness, and exports: the case of Senegal", Journal of African Economies, 11 (2), Oxford University Press, Oxford, pp. 219-248.

Moreno-Brid, Juan Carlos (1999), “Mexico's Economic Growth and the Balance of Payments Constraint: a Cointegration Analysis", International Review of Applied Economics, 13 (2), Routledge, Oxford, pp. 149-159.

Mortimore, Michael, Sebastián Vergara y Jorge Katz (2001), "La competitividad internacional y el desarrollo nacional: implicancias para la política de inversión extranjera directa en América Latina”, Documento de Trabajo 107, CEPAL, México.

Padilla, Ramón y Míriam Juárez (2006), "Efectos de la capacitación en la competitividad de la industria manufacturera", Serie Estudios y Perspectivas, CePal, México.

Patterson, Kerry (2000), An Introduction to Applied Econometrics: A Time Series Approach, MacMillan, Londres.

Perron, Pierre y Serena Ng (1996), "Useful modifications to unit root tests with dependent error and their local asymptotic properties", Review of Economic Studies, 63, Blackwell Publishing, Londres, pp. 435-465.

Pesaran, Hashem y Yongcheol Shin (1998), "Impulse response analysis in linear multivariate models", Economic Letters, 58, Elsevier, Ámsterdam, pp. 17-29.

Pugel, Thomas (2004), Economía internacional, McGraw Hill Interamericana, Madrid. 
Reinhart, Carmen (1995), "Devaluation, relative prices, and international trade: evidence from developing countries", IMF Staff Papers, 42 (2), Fondo Monetario Internacional, Washington, pp. 290-312.

Riedel, James (1988), "The demand for LDC exports of manufactures: estimates from Hong Kong", The Economic Journal, 98, Royal Economic Society, Saint Andrews, Fife, pp. 138-148.

Schwert, William (1989), "Testing for unit roots: a Monte Carlo investigation", Journal of Business and Economic Statistics, 7, American Statistical Association, Alexandria, Virginia, pp. 147-159.

Senhadji, Abdelhak y Claudio Montenegro (1998), "Time-series analysis of export demand equations: a cross-country analysis", IMF Working Paper 149, Fondo Monetario Internacional, Washington, pp. 1-29.

Sims, Christopher (1980), "Macroeconomics and reality", Econometrica, 48 (1), The Econometric Society, Cleveland, Ohio, pp. 1-49.

Wagner, Joachim (2007), "Exports and productivity: a survey of the evidence from firm-level data", The World Economy, 30 (1), Backwell Publishing, Oxford, pp. 60-82.

Walsh, Carl (2003), Monetary theory and policy, The мIт Press, Cambridge.

Recibido: 29 de julio de 2009. Reenviado: 11 de mayo de 2010. Aceptado: 17 de mayo de 2010.

Víctor Manuel Cuevas-Ahumada. Es doctor en economía por la Universidad de Missouri-Columbia, Estados Unidos. Es licenciado en administración por la Universidad Autónoma Metropolitana, Azcapotzalco (UAM-A); cuenta con una maestría en economía y política internacional por el Centro de Investigación y Docencia Económicas (CIDE), y una maestría en economía por la Universidad de Missouri-Columbia. Es miembro del Sistema Nacional de Investigadores (SNI), nivel I, y profesorinvestigador del Departamento de Economía de la UAM-A. Sus líneas de investigación se centran en econometría, sector manufacturero, política 
fiscal y finanzas internacionales. Entre sus publicaciones recientes destacan: "The short-term effects of fiscal policy in Mexico", Estudios Económicos, 24 (1), El Colegio de México, México, pp. 109-144 (2009); "Inflation, output and economic policy in Mexico", Investigación Económica, 67 (265), unam, México, pp. 85-120 (2008); "Inflación, crecimiento y política macroeconómica en Brasil y México: una investigación teóricoempírica", Revista EconoQuantum, 4 (2), Universidad de Guadalajara, Zapopan, pp. 35-78 (2008). 